\title{
Dynamics of concentrated suspensions of non-colloidal particles in Couette flow
}

\author{
KYONGMIN YEO AND MARTIN R. MAXEY† \\ Division of Applied Mathematics, Brown University, Providence, RI 02912, USA
}

(Received 18 August 2009; revised 11 November 2009; accepted 11 November 2009)

Fully three-dimensional numerical simulations of concentrated suspensions of $O(1000)$ particles in a Couette flow at zero Reynolds number are performed with the goal of determining the wall effects on concentrated suspensions of non-colloidal particles. The simulations, based on the force-coupling method, are performed for $0.2 \leqslant$ $\phi \leqslant 0.4$ and $10<L_{y} / a<30$, where $\phi$ denotes the volume fraction and $L_{y}$ and $a$ are, respectively, the channel height and the particle radius. It is shown that the suspensions can be divided into three regions depending on the microstructures; the wall region where a structured particle layering is dominant, the core region in which the suspension field is quasi-homogeneous, and the buffer region which shows the characteristics of both the particle layer and the shear structure. The width of the inhomogeneous region (wall and buffer) is a function of $\phi$ and not sensitive to $L_{y} / a$, once $L_{y} / a$ is larger than a threshold. Rheological properties in the inhomogeneous and quasi-homogeneous regions are investigated. The particle stresses are compared with previous rheological models.

\section{Introduction}

Due to its relevance to many engineering processes as well as biological applications, such as materials processing, waste treatment, particle coating and blood flow, the rheology of suspensions of spheres at low Reynolds numbers has been extensively studied (Stickel \& Powell 2005). Since the pioneering studies of Einstein (Einstein 1956) and Batchelor (Batchelor 1970; Batchelor \& Green 1972a,b), the rheological behaviour of dilute and semidilute suspensions, in which the lubrication interactions are neglected or limited to particle doublets, is well understood. In concentrated suspensions, however, not only long-range far-field hydrodynamics but also shortrange hydrodynamic (lubrication forces) and non-hydrodynamic interactions (surface roughness or electrostatic force) play an important role. This results in the complex rheological and non-Newtonian behaviour of concentrated suspensions of noncolloidal particles in shear flow, as reported by Brady \& Morris (1997); Zarraga et al. (2000); Stickel \& Powell (2005), amongst others.

The complex nature of concentrated suspensions makes it challenging to construct a theoretical model. Most of our current understanding of non-colloidal suspensions comes from experimental and numerical observations. In providing detailed information about the microstructure as well as the rheological properties,

$\dagger$ Email address for correspondence: maxey@cfm.brown.edu 
numerical simulations greatly contribute to our understanding on concentrated suspensions in ways not always available in experiments. The most widely used numerical method to study concentrated suspensions is the Stokesian dynamics (SD) method (Brady \& Bossis 1988; Brady 2001). In the case of homogeneous sheared suspensions in which periodic boundary conditions are used for all three-directions, modelling an infinite domain, SD has been used for a wide range of volume fractions to investigate the rheology and microstructure (Sierou \& Brady 2002) and shearinduced diffusion (Drazer et al. 2002; Sierou \& Brady 2004).

Although the understanding of concentrated suspensions in the unbounded domain is now much more advanced, much less is known about the behaviour of suspensions in the wall-bounded domain, where the presence of a solid boundary makes the characteristics of the suspension dramatically different. There have been several observations on the distinctive mechanisms in the wall-bounded suspensions, such as shear-induced migration of particles (Leighton \& Acrivos 1987; Nott \& Brady 1994; Lyon \& Leal 1998), apparent wall-slip (Jana et al. 1995), particle structuring (Komnik et al. 2004) and swapping of particle trajectories (Zurita-Gotor et al. 2007). There have been a few numerical studies on the wall-bounded suspensions using the SD, where a wall is replaced with a chain of fixed spheres (Nott \& Brady 1994; Singh \& Nott 2000). Due to the high computational cost, dynamic simulations in the wall-bounded flows have been limited to a monolayer simulation with $O(10)$ particles. Bossis et al. (1991) and Swan \& Brady (2007) have extended SD for particle-wall interactions using image methods (Blake \& Chwang 1974). However, dynamic simulations of concentrated suspensions using these modification have not been reported yet.

There are only a few three-dimensional simulations of concentrated suspensions in Couette flow. Most of the simulations are performed using the lattice-Boltzmann (LB) method as developed by Nguyen \& Ladd (2002). Kromkamp et al. (2006) performed both two- and three-dimensional LB simulations of concentrated suspensions in a Couette flow. They showed that the wall structuring in two-dimensional simulation differs from the three-dimensional results. They observed dependency of some results on the computational resolution and ascribed the dependency to the ragged particle surface in the LB method. Kulkarni \& Morris (2008) performed three-dimensional LB simulations for the volume fraction up to 0.3 in a Couette flow to investigate the finite-Reynolds-number effects on the suspension rheology.

The force-coupling method (FCM) was developed by Maxey \& Patel (2001) to simulate a large system of particles in suspension. For example, using FCM, Dance \& Maxey (2003b) performed numerical simulations of sedimentation flows bounded by top and bottom walls with up to 10000 particles. In the FCM, far-field hydrodynamic interaction between particles is calculated by solving the Stokes equations (Lomholt \& Maxey 2003). As in LB method, the wall boundary is treated naturally by imposing the no-slip boundary condition.

The FCM for Stokes flows is a far-field approximation of the grand-mobility matrix (Yeo \& Maxey, 2010). FCM does not represent the exact solution if the distance between two particles is closer than $r / a<2.4$ (Lomholt \& Maxey 2003), in which $r$ and $a$ denote the particle centre-to-centre distance and the particle radius, respectively. Most FCM simulations have been performed in the semidilute regime, where the volume fraction $\phi<0.2$. Dance \& Maxey (2003a) suggested a fast lubrication correction method to resolve the lubrication effects between particles, summing pairwise interactions within a mobility formulation. This is adequate for low volume fractions, but more recently Yeo \& Maxey (2010) developed a more robust lubrication correction method for the simulations of concentrated suspensions. 
The main goals of this paper are to extend the lubrication correction developed in Yeo \& Maxey (2010) to the wall-bounded flows and investigate the effects of the wall on the suspension rheology. The present simulations are among the first computational results on the dynamics of concentrated Stokes suspensions in a Couette flow with a full representation of no-slip conditions at a rigid wall. It is shown that particle layers are formed near the wall due to the strong particle-wall lubrication interactions. As a result of the particle layering, the rheological properties such as the relative viscosity and the normal stresses are found to be functions of $L_{y} / a$ in which $L_{y}$ is the channel height. At high volume fractions the suspension can be divided into three regions depending on the microstructure; the wall region where particle layering is dominant, the core region in which suspension behaves similarly to homogeneous suspensions and the buffer region in which the microstructure shows the characteristics of both the shear structure and the particle layer.

In $\S 2$, brief review of the FCM and the particle-wall lubrication models are presented. The numerical results for a single particle in a channel flow are compared with the previous results in $\S 3$. Section 4 provides the main results of the numerical simulations in the Couette flow.

\section{Force-coupling method in the wall-bounded flows}

\subsection{Review of force-coupling method and lubrication correction}

In the FCM, the delta function of the standard singular multipole expansion is replaced by a finite, smoothly varying function $\Delta(\boldsymbol{x}-\boldsymbol{Y})$ and the multipole expansion is truncated at the dipole level (Maxey \& Patel 2001; Lomholt \& Maxey 2003). The equation of fluid motion in the FCM is

$$
\frac{\partial p}{\partial x_{i}}=\mu \nabla^{2} u_{i}+\sum_{n=1}^{N_{p}}\left\{F_{i}^{n} \Delta_{M}\left(\boldsymbol{x}-\boldsymbol{Y}^{n}\right)+G_{i j}^{n} \frac{\partial}{\partial x_{j}} \Delta_{D}\left(\boldsymbol{x}-\boldsymbol{Y}^{n}\right)\right\},
$$

where $p$ is pressure, $\mu$ is viscosity, $\boldsymbol{u}$ is fluid velocity, $\boldsymbol{Y}^{n}$ is the location of a particle centre and $F_{i}$ and $G_{i j}$ are the force monopole and force dipole moments, respectively. The FCM force envelopes $\Delta_{M}$ and $\Delta_{D}$ are given by

$$
\begin{aligned}
& \Delta_{M}(\boldsymbol{x})=\frac{1}{\left(2 \pi \sigma_{M}^{2}\right)^{3 / 2}} \exp \left(-\frac{\boldsymbol{x}^{2}}{2 \sigma_{M}^{2}}\right), \\
& \Delta_{D}(\boldsymbol{x})=\frac{1}{\left(2 \pi \sigma_{D}^{2}\right)^{3 / 2}} \exp \left(-\frac{\boldsymbol{x}^{2}}{2 \sigma_{D}^{2}}\right),
\end{aligned}
$$

in which $\sigma_{M}=a / \sqrt{\pi}$ and $\sigma_{D}=a /(6 \sqrt{\pi})^{1 / 3}$. As a consequence of the finite envelope, FCM essentially resolves too the potential dipole and the potential quadrupole flows associated with the force monopole and force dipole. See Lomholt \& Maxey (2003) for detailed discussions.

The force monopole represents the force exerted on the fluid by a particle resulting, for example, from the force due to gravity, short-range inter-particle forces, Brownian motion or electromagnetic forces on the particle. The force dipole consists of a symmetric part, $S_{i j}$ and an antisymmetric part $T_{i j}$. $S_{i j}$ corresponds to the stresslet and $T_{i j}$ is the couplet. $T_{i j}$ is related with the external torque $T^{e x t}$ as

$$
T_{i j}=\frac{1}{2} \epsilon_{i j k} T_{k}^{e x t} .
$$


$S_{i j}$ is obtained from the rigidity constraint on the particle (Lomholt \& Maxey 2003),

$$
\int e_{i j} \Delta_{D}\left(\boldsymbol{x}-\boldsymbol{Y}^{n}\right) \mathrm{d}^{3} \boldsymbol{x}=0
$$

in which $e_{i j}$ denotes the strain rate,

$$
e_{i j}=\frac{1}{2}\left(\frac{\partial u_{i}}{\partial x_{j}}+\frac{\partial u_{j}}{\partial x_{i}}\right) .
$$

The velocity of each particle $\boldsymbol{V}^{n}$ is obtained by the weighted volume integral of $\boldsymbol{u}(\boldsymbol{x})$ (Maxey \& Patel 2001),

$$
\boldsymbol{V}^{n}=\int \boldsymbol{u}(x) \Delta_{M}\left(\boldsymbol{x}-\boldsymbol{Y}^{n}\right) \mathrm{d}^{3} \boldsymbol{x} .
$$

Similarly, the angular velocity of each particle $\boldsymbol{\Omega}^{n}$ is

$$
\Omega_{i}^{n}=\frac{1}{2} \int \epsilon_{i j k} \frac{\partial u_{k}}{\partial x_{j}} \Delta_{D}\left(\boldsymbol{x}-\boldsymbol{Y}^{n}\right) \mathrm{d}^{3} \boldsymbol{x} .
$$

These relations ensure that the flow satisfies the corresponding reciprocal theorem and captures to a good approximation the Faxen corrections for non-uniform flows (Happel \& Brenner 1965).

In the FCM, long-range multi-body interactions are computed by solving the Stokes equations (2.1). In Yeo \& Maxey (2010), it is shown that the force-coupling procedure is equivalent to solving a mobility problem. Symbolically, the force-coupling procedure can be written as

$$
\left[\begin{array}{c}
\mathscr{V}-\mathscr{V}^{\infty} \\
\boldsymbol{E}^{\infty}
\end{array}\right]=\boldsymbol{M}^{F C M}\left[\begin{array}{c}
\mathscr{F} \\
\boldsymbol{S}
\end{array}\right]=\left[\begin{array}{cc}
\boldsymbol{M}_{\mathscr{F} \mathscr{V}} & \boldsymbol{M}_{S \mathscr{V}} \\
-\boldsymbol{M}_{\mathscr{F} E} & -\boldsymbol{M}_{S E}
\end{array}\right]\left[\begin{array}{c}
\mathscr{F} \\
\boldsymbol{S}
\end{array}\right],
$$

in which $\boldsymbol{M}^{F C M}$ is the FCM grand mobility matrix and a submatrix $\boldsymbol{M}_{A B}$ is a mobility matrix to calculate a value $B$ from a given coefficient $A$. $\mathscr{V}$ is the $\left(6 N_{p}\right)$ vector of the translational and angular velocities, while $\mathscr{F}$ is the $\left(6 N_{p}\right)$ vector containing the monopole coefficients and torques. $S$ is a $\left(5 N_{p}\right)$ vector consisting of the five independent stresslet coefficients, $S_{11}, S_{12}, S_{13}, S_{22}, S_{23}$. Similarly, $\mathscr{V}^{\infty}$ is the $\left(6 N_{p}\right)$ vector of the translational and angular velocities of the imposed external flow field and $\boldsymbol{E}^{\infty}$ is a $\left(5 N_{p}\right)$ vector of the five independent components of the corresponding strain rate of the imposed field. The FCM grand mobility matrix provides a far-field approximation of the exact mobility problem.

On the right-hand side of (2.9), $\mathscr{F}$ is given while $S$ is determined by the rigidity constraint (2.5),

$$
\boldsymbol{E}^{\infty}+\boldsymbol{M}_{\mathscr{F} E} \mathscr{F}=-\boldsymbol{M}_{S E} \boldsymbol{S}
$$

The matrix $-\boldsymbol{M}_{S E}$ is symmetric and positive semidefinite. The system of equations thus can be solved by using a conjugate gradient method.

In order to incorporate the near-field interactions, Yeo \& Maxey (2010) employed the pair-wise additivity of the lubrication interactions to the grand resistance matrix (Durlofsky et al. 1987; Brady \& Bossis 1988). FCM with the lubrication interactions included then results in the system of equations

$$
\left[\begin{array}{c}
\boldsymbol{M}_{\mathscr{F} \mathscr{V}}^{\mathscr{F}^{t o t}} \\
\boldsymbol{M}_{\mathscr{F} E} \mathscr{F}^{t o t}+\boldsymbol{E}^{\infty}
\end{array}\right]=\left[\begin{array}{cc}
\mathscr{R}^{-1}+\boldsymbol{M}_{\mathscr{F} \mathscr{V}} & -\boldsymbol{M}_{S \mathscr{V}} \\
\boldsymbol{M}_{\mathscr{F} E} & -\boldsymbol{M}_{S E}
\end{array}\right]\left[\begin{array}{c}
\mathscr{F} l u b \\
\boldsymbol{S}^{t o t}
\end{array}\right],
$$


in which $\mathscr{R}$ is the lubrication correction to the resistance matrix relating the translational and angular velocities to the hydrodynamic forces and torques. $\mathscr{R}$ is constructed as the sum of the particle-pair resistance matrices $\mathscr{R}_{2 B}=\mathscr{R}_{2 B}^{E}-\mathscr{R}_{2 B}^{F C M}$, where $\mathscr{R}_{2 B}^{E}$ and $\mathscr{R}_{2 B}^{F C M}$ denote the exact and FCM two-body resistance matrices, respectively, for individual pairs of particles. $\mathscr{F}^{\text {tot }}$ and $\boldsymbol{S}^{\text {tot }}$ are given as

$$
\begin{aligned}
\mathscr{F}^{t o t} & =\mathscr{F}+R_{E \mathscr{F}} \boldsymbol{E}^{\infty}, \\
S^{t o t} & =S+R_{E S} \boldsymbol{E}^{\infty}-R_{\mathscr{V} S}\left(\mathscr{V}-\mathscr{V}^{\infty}\right),
\end{aligned}
$$

in which $R_{A B}$ is a resistance matrix relating $A$ to $B$.

The matrix on the right-hand side of (2.11) is the FCM grand mobility matrix with the lubrication correction $\mathscr{R}^{-1}$. By use of a preconditioned conjugate gradient method, $\mathscr{R}^{-1}$ in (2.11) can be replaced by a recursive formula which consists of a summation of two vectors. Hence, the computational cost is largely determined by the choice of the Stokes solver to solve (2.1). Once the relevant lubrication forces and torques, given by $\mathscr{F}^{l u b}$, are determined, then the translational and angular velocities are computed from either

$$
\mathscr{V}=\mathscr{V}^{\infty}+\mathscr{R}^{-1} \mathscr{F}^{l u b},
$$

or

$$
\mathscr{V}=\mathscr{V}^{\infty}+\boldsymbol{M}_{\mathscr{F} \mathscr{V}}\left(\mathscr{F}^{t o t}-\mathscr{F}^{l u b}\right)+\boldsymbol{M}_{S \mathscr{V}} \boldsymbol{S}^{\text {tot }} .
$$

Further details are given in Yeo \& Maxey (2010). Note that FCM calculates $\boldsymbol{S}^{\text {tot }}$ and not the stresslet $S$ in the computation. The stresslet is calculated from (2.13) as a post-processing step.

\subsection{Modification of the force-coupling method envelopes near a wall}

For wall-bounded flows, (2.1) is solved numerically with the appropriate no-slip boundary conditions for rigid walls to obtain the incompressible Stokes flow field $\boldsymbol{u}$. This gives the far-field flow and then, in addition to the viscous lubrication forces between particles, particle-wall lubrication interactions need to be accounted for as described next in $\S 2.3$. The force envelopes, $\Delta_{M}$ and $\Delta_{D}$, while narrowly confined to each particle do extend beyond the physical size of the particle. As a result, it is possible that when a particle is close to a wall the envelope overlaps the boundary. Similarly, in evaluating the integrals for the particle velocity (2.7) or for the rate of strain (2.5) and the angular velocity (2.8) the range of integration goes outside of the physical flow domain. In previous simulations of wall-bounded flows based on FCM (Liu et al. 2002, 2004; Lomholt \& Maxey 2003; Dance \& Maxey 2003b), if a particle is close to a wall then the FCM envelope is truncated at the wall boundary in (2.1) and integration is confined to the physical flow domain.

For a particle in contact with a rigid boundary, the truncated volume in (2.7) is approximately $3.8 \%$ of the total and similarly for (2.5) is $1.4 \%$. The effects of this truncation procedure can be included and accounted for at low volume fractions in the estimates of the particle-wall lubrication forces (Dance \& Maxey 2003a). This procedure is self-consistent. However, it presents a minor problem for evaluating results directly related to an imposed external shear flow.

As an illustration, consider a flow bounded by a rigid wall at $x_{2}=0$ so that the flow domain $\boldsymbol{\Omega}^{D}$ is the semi-infinite region $\boldsymbol{\Omega}^{D}=(-\infty, \infty) \times(0, \infty) \times(-\infty, \infty)$ and the wall is located on the boundary $\partial \boldsymbol{\Omega}^{D}$. A particle in a linear shear flow $\boldsymbol{u}^{\infty}=\left(\dot{\gamma} x_{2}, 0,0\right)^{T}$, 
in which $\dot{\gamma}$ is the shear rate, would usually give the result from FCM that

$$
\boldsymbol{V}^{\infty}=\boldsymbol{u}^{\infty}(\boldsymbol{Y})=\int_{\boldsymbol{I}_{n}(\boldsymbol{Y})} \boldsymbol{u}^{\infty}(\boldsymbol{x}) \Delta_{M}(\boldsymbol{x}-\boldsymbol{Y}) \mathrm{d}^{3} \boldsymbol{x} .
$$

The domain of numerical integration is $\boldsymbol{I}_{\boldsymbol{n}}(\boldsymbol{Y})=\left\{\boldsymbol{x}: \boldsymbol{x} \in \mathbb{R}^{3}, \quad|\boldsymbol{x}-\boldsymbol{Y}|<a \times n\right\}$, representing a sphere of larger radius $n a$ where $n$ is a positive real number. In order to obtain full accuracy, $n$ is usually chosen such that $n \geqslant 2.5$. If a particle is close to a wall such that $\boldsymbol{I}_{n}(\boldsymbol{Y}) \nsubseteq \boldsymbol{\Omega}^{D}$, then the FCM envelope is truncated at the wall boundary, i.e. the integration is performed only in $\boldsymbol{I}_{n}(\boldsymbol{Y}) \cap \boldsymbol{\Omega}^{D}$. This results in $\boldsymbol{V}^{\infty} \neq \boldsymbol{u}^{\infty}(\boldsymbol{Y})$.

As a remedy to this problem, the FCM envelope is modified for particles near a wall $\left(Y_{2}<a \times n\right)$ and an image envelope is introduced. That is for the force monopole term,

$$
\Delta_{M}^{\text {Wall }}(\boldsymbol{x}-\boldsymbol{Y})=\Delta_{M}(\boldsymbol{x}-\boldsymbol{Y})-\Delta_{M}\left(\boldsymbol{x}-\boldsymbol{Y}^{\text {Img }}\right),
$$

in which $\boldsymbol{Y}^{I m g}=\left(Y_{1},-Y_{2}, Y_{3}\right)^{T}$. Similarly, the dipole envelope is given as,

$$
\Delta_{D}^{\text {Wall }}(\boldsymbol{x}-\boldsymbol{Y})=\Delta_{D}(\boldsymbol{x}-\boldsymbol{Y})+\Delta_{D}\left(\boldsymbol{x}-\boldsymbol{Y}^{I m g}\right) .
$$

The modified wall envelopes ensure that

$$
\boldsymbol{V}^{\infty}=\boldsymbol{u}^{\infty}(\boldsymbol{Y})=\int_{\boldsymbol{I}_{n}(\boldsymbol{Y}) \cap \boldsymbol{\Omega}^{D}} \boldsymbol{u}^{\infty}(\boldsymbol{x}) \Delta_{M}^{\text {Wall }}(\boldsymbol{x}-\boldsymbol{Y}) \mathrm{d}^{3} \boldsymbol{x},
$$

and

$$
E_{i j}^{\infty}=e_{i j}^{\infty}(\boldsymbol{Y})=\int_{\boldsymbol{I}_{n}(\boldsymbol{Y}) \cap \boldsymbol{\Omega}^{D}} e_{i j}^{\infty}(\boldsymbol{x}) \Delta_{D}^{\text {Wall }}(\boldsymbol{x}-\boldsymbol{Y}) \mathrm{d}^{3} \boldsymbol{x},
$$

as well as ensuring that the particle angular velocity matches the fluid vorticity (2.8) for the imposed external flow. This modified procedure is also self-consistent. Coincidentally, the image envelope $\Delta_{M}^{\text {Wall }}$ would correspond to the lowest order image Stokeslet as given by Blake \& Chwang (1974), where they apply image methods to solve for Stokes flow in the presence of a rigid boundary. However, the reasons for the formulation of the wall-envelopes are different and the underlying numerical solution to (2.1) already ensures that the no-slip conditions are satisfied.

\subsection{Particle-wall lubrication correction}

Close to a rigid no-slip boundary, additional viscous lubrication corrections are required for the motion of a particle relative to the wall boundary. This modification for FCM is given by Dance \& Maxey (2003a) in the simpler context of dilute suspensions. We summarize briefly, and with more general notation, the steps for adding these corrections in the resistance matrix.

The resistance relation of a single particle moving in a flow near a wall is

$$
\left[\begin{array}{c}
\boldsymbol{F} \\
\boldsymbol{T}
\end{array}\right]=\mu\left[\begin{array}{ccc}
\boldsymbol{A} & \boldsymbol{B} & \boldsymbol{G} \\
\boldsymbol{B}^{T} & \boldsymbol{C} & \boldsymbol{H}
\end{array}\right]\left[\begin{array}{c}
\boldsymbol{V}-\boldsymbol{V}^{\infty}(\boldsymbol{Y}) \\
\boldsymbol{\Omega}-\boldsymbol{\Omega}^{\infty}(\boldsymbol{Y}) \\
-\boldsymbol{E}^{\infty}
\end{array}\right] .
$$


Following the notation in Kim \& Karrila (1991), the resistance tensors are

$$
\begin{aligned}
A_{i j} / 6 \pi a & =X^{A} d_{i} d_{j}+Y^{A}\left(\delta_{i j}-d_{i} d_{j}\right), \\
B_{i j} / 6 \pi a^{2} & =Y^{B} \epsilon_{j i k} d_{k}, \\
C_{i j} / 8 \pi a^{3} & =X^{C} d_{i} d_{j}+Y^{C}\left(\delta_{i j}-d_{i} d_{j}\right), \\
G_{k i j} / 6 \pi a^{2} & =Y^{G}\left(d_{i} \delta_{j k}+d_{j} \delta_{i k}-2 d_{i} d_{j} d_{k}\right), \\
H_{k i j} / 8 \pi a^{3} & =Y^{H}\left(\epsilon_{i k l} d_{l} d_{j}+\epsilon_{j k l} d_{l} d_{i}\right),
\end{aligned}
$$

in which $\boldsymbol{d}$ is the unit vector from the particle centre to a wall. The exact, theoretical values for the various wall resistance functions are summarized in the Appendix, where they are given as asymptotic series in the gap width $a \epsilon$ between the particle and the wall.

Second, the non-singular estimates of the corresponding wall resistance functions from FCM are determined numerically for a single particle. This computation is done in the domain $\Omega^{D}=\left(0, L_{x}\right) \times\left(0, L_{y}\right) \times\left(0, L_{z}\right)$, where periodic boundary conditions are applied in the $x_{1}$ and $x_{3}$ directions with the no-slip boundary conditions $(\boldsymbol{u}=0)$ on $x_{2}=0$ and $L_{y}$. A Fourier spectral method is used in the horizontal $\left(x_{1}, x_{3}\right)$ directions and a spectral element method is employed in the vertical direction $\left(x_{2}\right)$ (see appendix in Dance \& Maxey 2003b). The Stokes equations are solved by applying the Uzawa algorithm. The Uzawa algorithm provides a saddle decoupling of the velocity and pressure fields, which is computationally more efficient than solving the fully coupled system (for details, see Maday et al. 1993; Karniadakis \& Sherwin 2005). In order to estimate the FCM resistance functions, the numerical simulations were performed in a large channel, $L_{x} / a=L_{y} / a=L_{z} / a=60$, so as to minimize the effects of the periodicity and the presence of the upper wall. A sphere is located at $L_{x} / a=L_{z} / a=30$. The hydrodynamic drag and torque were computed, varying the distance from the lower wall for the different configurations of particle motion or flow. The results were then matched to a regular asymptotic expansion for the FCM resistance function for small values of the gap $a \epsilon$ between the particle and the lower wall. The FCM resistance functions used in the simulations are also given in the Appendix.

The lubrication correction is implemented in the same way as described in Yeo \& Maxey (2010). First, $\mathscr{R}$ is constructed by summing all the particle-pair interactions. Since the lubrication force arises from the relative motion between particles, $\mathscr{R}_{2 B}$ is written in the relative velocity formulation (Nguyen \& Ladd 2002; Cichocki et al. 1999). Then, the resistance tensors for the particle-wall lubrication are added to $\mathscr{R}$.

\section{Single particle in a channel}

We first illustrate the methods described above by applying them to the motion of a single particle in a channel bounded by two parallel planar walls. The first example is of a spherical particle moving perpendicular to the walls under the action of an external force but in the absence of any other flow. The configuration of the channel geometry is shown in figure 1. Numerical results for the non-dimensional resistance coefficient $\lambda^{t}$, defined by

$$
\lambda^{t}=\frac{F_{2}}{6 \pi \mu a V_{2}},
$$

have been obtained previously by Ganatos, Weinbaum \& Preffer (1980) using a series solution and boundary collocation scheme for a channel with infinite planar walls. 


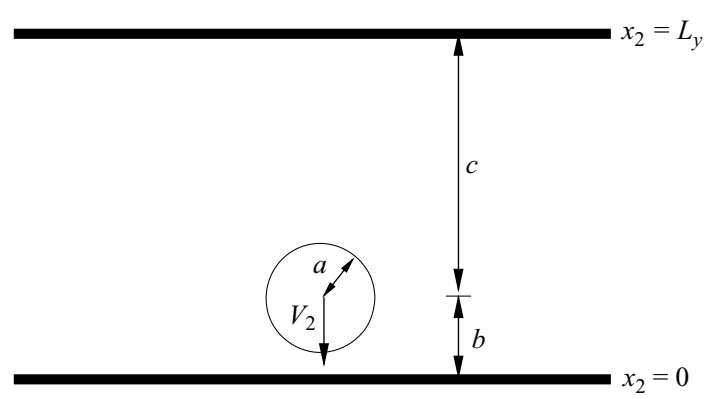

FIGURE 1. Geometry for a sphere placed between two walls.

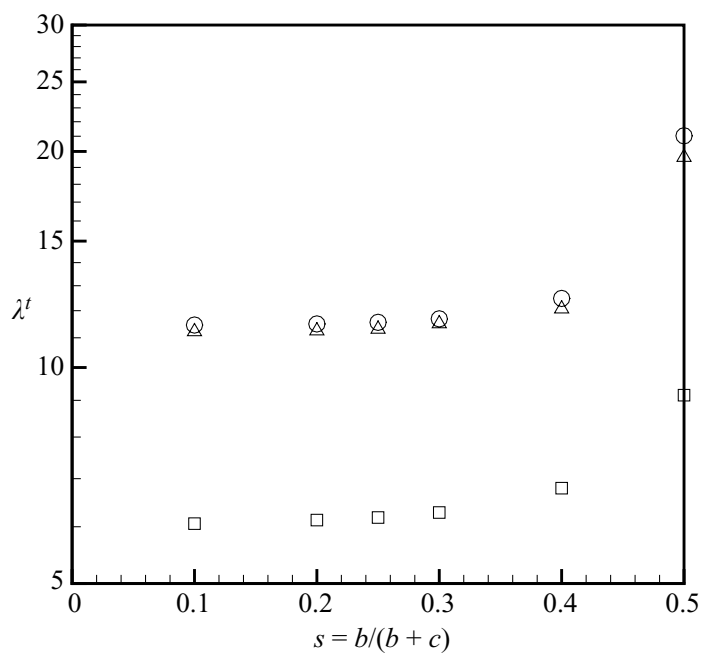

Figure 2. Drag coefficient $\lambda^{t}$ from $\bigcirc$, Ganatos et al. (1980); $\triangle$, FCM with wall lubrication; $\square$, FCM with monopole and dipole.

The corresponding FCM simulations are performed for a periodic channel, where $L_{x} / a=L_{z} / a=60$. The height of the channel $L_{y}$ is varied according to the parameter $s=b /(b+c)$ used by Ganatos et al. (1980). The gap between the particle and the wall is $0.1 a$ so that $b / a=1.1$.

A comparison of the results for $\lambda^{t}$ from the two different approaches is shown in figure 2. Also shown are the values from FCM without the lubrication correction, which are roughly half the total for $\lambda^{t}$. This configuration is the most challenging to calculate with FCM, the gap being larger than at which near-field lubrication forces would dominate, and tests the matching procedure for the near-field and far-field conditions. In general the agreement of the results is very good, with differences of $2 \%$ or less for the most part. For the larger values of $s$ the discrepancies are larger. For $s=0.5$, the channel height is only $2.2 a$ and the calibration procedures used to obtain the FCM wall resistance functions, which were based on widely separated channel walls are no longer appropriate. The results for the Couette flow suspension presented in $\S 4$ correspond to $s \leqslant 0.11$.

The second example is for the translational velocity of a force-free and torque-free sphere in a Couette flow. The computational domain here is $L_{x}=30, L_{z}=20$ and $L_{y}=10$ and the particle radius $a=1$. The upper wall is moving in the $x_{1}$ direction with the velocity $V_{u p p}=-1.0$, while the lower wall velocity is $V_{l o w}=1.0$. Figure 3 shows the 


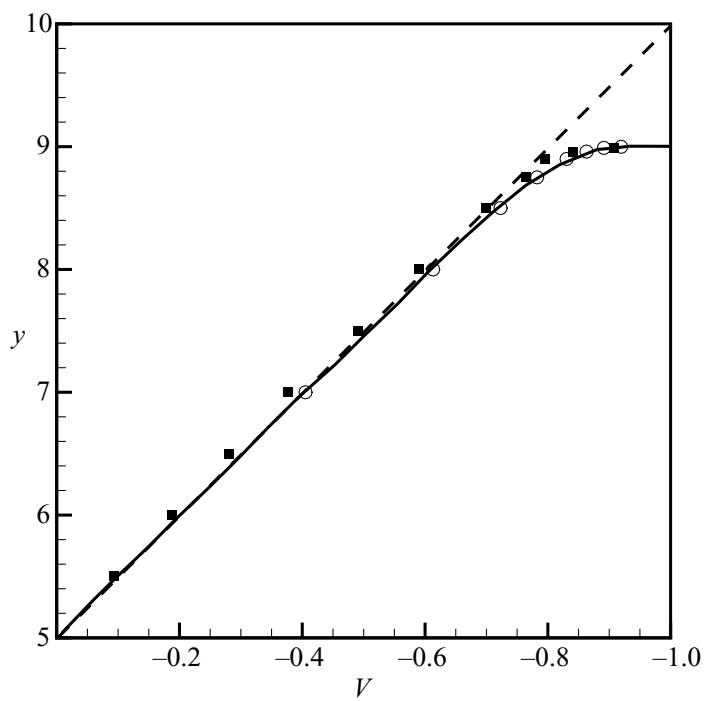

FIGURE 3. Translational velocity of a sphere $V$ in a Couette flow: solid line, Ganatos et al. (1982); $\mathbf{n}$, Singh \& Nott (2000); O, present FCM.

translational velocity of a sphere centred at various $y$ locations, close to the upper wall at $y=10$. The FCM results are compared with the boundary collocation results of Ganatos, Weinbaum \& Preffer (1982) and the SD simulation by Singh \& Nott (2000). The results in Ganatos et al. (1982) are used as the reference. In the FCM simulations, the lubrication corrections are used when $y / a>8.8$, that is when the gap between the particle and the wall is less than $0.2 a$. In the region where the far field solution plays the dominant role, the FCM results match those of Ganatos et al. (1982) without any corrections. In the near-wall region, FCM gives more accurate results than the SD results reported in Singh \& Nott (2000). In the latter, a rigid wall was represented as a layer of fixed spherical 'wall particles' rather than a smooth wall with no-slip boundary conditions. Further, the domain size was smaller, with periodic dimensions $L_{x} / a=L_{z} / a=14$.

\section{Couette flow suspensions}

The main focus of this study is on the dynamics of concentrated suspensions of non-Brownian particles in Couette flow, where the presence of the rigid walls has a significant effect on the particle motion. To this end, numerical simulations are performed for three different volume fractions $(\phi=0.2,0.3,0.4)$ using the same numerical method described in $\S 2$. The computational domain in the horizontal directions is fixed $\left(L_{x} / a=30, L_{z} / a=20\right)$, while the channel height is varied to investigate the effect of the wall $\left(L_{y} / a=10,20,30\right)$. The number of Fourier modes in $x$ and $z$ directions are 96 and 64, respectively. The simulation parameters are shown in table 1 . The radius of a sphere $(a)$ and the shear rate $(\dot{\gamma})$ are fixed at reference values: $a=1, \quad \dot{\gamma}=1$. The upper wall is moving with the velocity $\boldsymbol{V}_{\text {wall }}=\left(\dot{\gamma} L_{y} / 2,0,0\right)^{T}$ and the lower wall is moving in the opposite direction with the same speed. The equation for the particle position,

$$
\frac{\mathrm{d} \boldsymbol{Y}^{n}}{\mathrm{~d} t}=\boldsymbol{V}^{n}
$$

is solved by the third-order Adam-Bashforth scheme. 


$\begin{array}{lccrcccc} & \phi & L_{y} & N_{y} & N_{p} & R_{\text {ref }} & F_{\text {ref }} & \mathrm{d} t \\ \text { C2S } & 0.2 & 10 & 4 & 285 & 2.01 & 100 & 2 \times 10^{-3} \\ \text { C3S } & 0.3 & 10 & 4 & 430 & 2.01 & 100 & 2 \times 10^{-3} \\ \text { C4S } & 0.4 & 10 & 4 & 567 & 2.01 & 100 & 2 \times 10^{-3} \\ \text { C2L } & 0.2 & 20 & 8 & 570 & 2.01 & 100 & 2 \times 10^{-3} \\ \text { C3L } & 0.3 & 20 & 8 & 860 & 2.01 & 100 & 2 \times 10^{-3} \\ \text { C4La } & 0.39 & 20 & 8 & 1122 & 2.01 & 100 & 2 \times 10^{-3} \\ \text { C4Lb } & 0.39 & 20 & 8 & 1122 & 2.004 & 100 & 2 \times 10^{-3} \\ \text { C4Lc } & 0.39 & 20 & 8 & 1122 & 2.001 & 1000 & 1 \times 10^{-3} \\ \text { C4H } & 0.4 & 30 & 12 & 1718 & 2.01 & 100 & 2 \times 10^{-3}\end{array}$

TABLE 1. Simulation parameters. $N_{y}$ is the number of elements in the vertical direction. Every elements has the same length $E_{L}=2.5$ and quadrature points $Q_{y}=9$. d $t$ is the computational time-step size.

When the separation distance between two particles becomes very small (less than $10^{-2} a$ ), non-hydrodynamic effects, such as surface roughness, residual Brownian forces and electrostatic forces, may play an important role. These effects will break the foreafter symmetry of particle interactions in Stokes flow. For example, Smart \& Leighton (1989) showed that the roughness of particles ranging from 43 to $6350 \mu \mathrm{m}$ in diameter is of the order of $10^{-2} a \sim 10^{-3} a$. Brady \& Morris (1997) showed the importance of these non-hydrodynamic forces on the non-Newtonian aspect of suspension flows. To model the non-hydrodynamic interactions, and specifically the effects of surface roughness of non-Brownian particles, a short-range contact force $\boldsymbol{F}_{P}^{i j}$ is used in the present simulations when the distance between particle $i$ and $j$ is less than a barrier cutoff distance $R_{r e f}$. The form of the contact force is chosen to be similar to Dance et al. (2004),

$$
\boldsymbol{F}_{P}^{i j}= \begin{cases}-6 \pi \mu \dot{\gamma} a^{2} F_{r e f}\left(\frac{R_{r e f}^{2}-|\boldsymbol{r}|^{2}}{R_{r e f}^{2}-4 a^{2}}\right)^{6} \frac{\boldsymbol{r}}{|\boldsymbol{r}|} & \text { if }|\boldsymbol{r}|<R_{r e f}, \\ 0 & \text { otherwise, }\end{cases}
$$

in which $\boldsymbol{r}=\boldsymbol{Y}^{i}-\boldsymbol{Y}^{j}$ and $F_{\text {ref }}$ is a constant. It has been discussed that, as long as the contact force is sufficiently short ranged, the detailed expression of the contact force does not change the simulation results significantly (Dratler \& Schowalter 1996; Marchioro \& Acrivos 2001). The effects of the roughness model or the contact force on the suspension rheology has been of interest and can be found in the literature (Da Cunha \& Hinch 1996; Brady \& Morris 1997; Meunier \& Bossis 2004; Abbas et al. 2006; Ingber et al. 2008).

Since the system is chaotic and ergodic, a time average is used to obtain statistics once the suspension flow reaches a statistically stationary state. The initial configurations are obtained by two different approaches; body-centred cubes with small random perturbations and a molecular dynamics simulation. It is observed that, once the suspension reaches a statistically stationary state, the statistics does not depend on the initial configuration. Simulations are performed for $\dot{\gamma} t \simeq 50$ to reach the stationary state. Then, the time average is performed over the period $T=100 \dot{\gamma} t$ except for C4Lc for which the simulation is performed until $T=$ $40 \dot{\gamma} t$. 


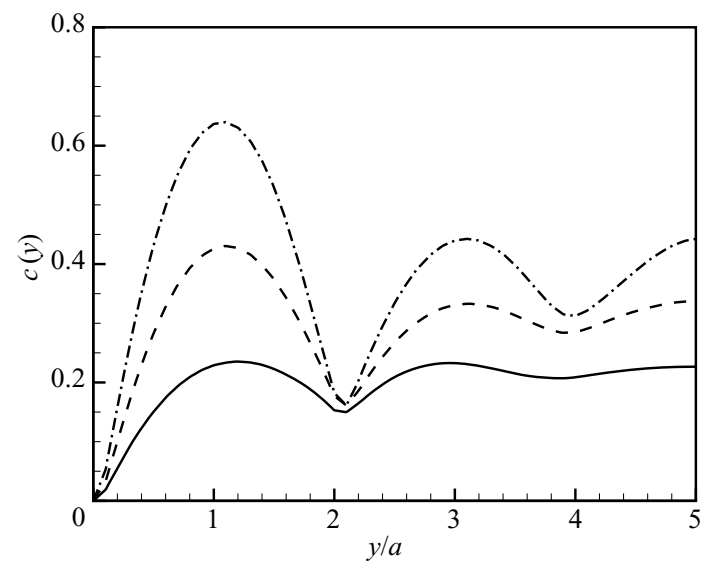

Figure 4. Concentration profiles for C2S (solid), C3S (dashed) and C4S (dash-dot).

\subsection{Wall effects on particle concentration}

In wall-bounded suspensions, once a particle moves close to a wall, the particle tends to stay near the wall for a long time due to the strong particle-wall lubrication force, which then results in the formation of stable particle layers near the wall. Using LB simulations, both Kromkamp et al. (2006) and Kulkarni \& Morris (2008) observed a pronounced local concentration peak near the wall at high volume fractions, which was not reported in the earlier simulations of coplanar particles in Couette flow (Singh \& Nott 2000). In this section, the concentration profiles and the twodimensional pair-distribution functions are presented to characterize the particle layering and the near-wall effects on suspensions.

The local concentration is defined as

$$
\left\langle c\left(x_{2}\right)\right\rangle=\frac{1}{L_{x} \times L_{z}}\left\langle\iint \chi(\boldsymbol{x}) \mathrm{d} x \mathrm{~d} z\right\rangle,
$$

in which $\chi(\boldsymbol{x})$ is an indicator function that is only non-zero if $\boldsymbol{x}$ is inside of a particle and $\langle\cdot\rangle$ denotes the ensemble (time) average.

Figure 4 shows the concentration profiles in the small domain $\left(L_{y} / a=10\right)$. Considering the symmetry, only half of the channel is drawn. The concentration profiles for $\mathrm{C} 2 \mathrm{~S}$ and $\mathrm{C} 3 \mathrm{~S}$ have a local peak in the wall region and become relatively flat away from the wall. On the other hand, the plateau is not observed in $\mathrm{C} 4 \mathrm{~S}$, indicating that the wall effect is dominant across the whole channel. As the layering in the wall region becomes stronger, it is seen that the ratio of the first local peak concentration near the wall to the concentration in the centre is an increasing function of $\phi$. For $\mathrm{C} 4 \mathrm{~S}$, the peak concentration in the particle layer is almost 1.5 times larger than that in the centre region. Consistent with Kromkamp et al. (2006), the location of the peak concentration moves towards the wall as $\phi$ increases. The local peaks are observed around 1.2, 1.1 and 1.08 for $\mathrm{C} 2 \mathrm{~S}, \mathrm{C} 3 \mathrm{~S}$ and $\mathrm{C} 4 \mathrm{~S}$, respectively. Due to the finite size of the particles, there is a particle depletion layer around $y / a \simeq 2.1$ even for the lowest volume fraction in the present simulation (C2S).

Further insight about the relative position of particles comes from the particle pair-distribution. The pair-distribution functions projected onto the velocity/velocitygradient plane, $g(\Delta x, \Delta y)$, and velocity-gradient/vorticity plane, $g(\Delta y, \Delta z)$, are shown in figure 5 for $\mathrm{C} 4 \mathrm{~S}$. The pair-distribution functions are obtained for the reference 

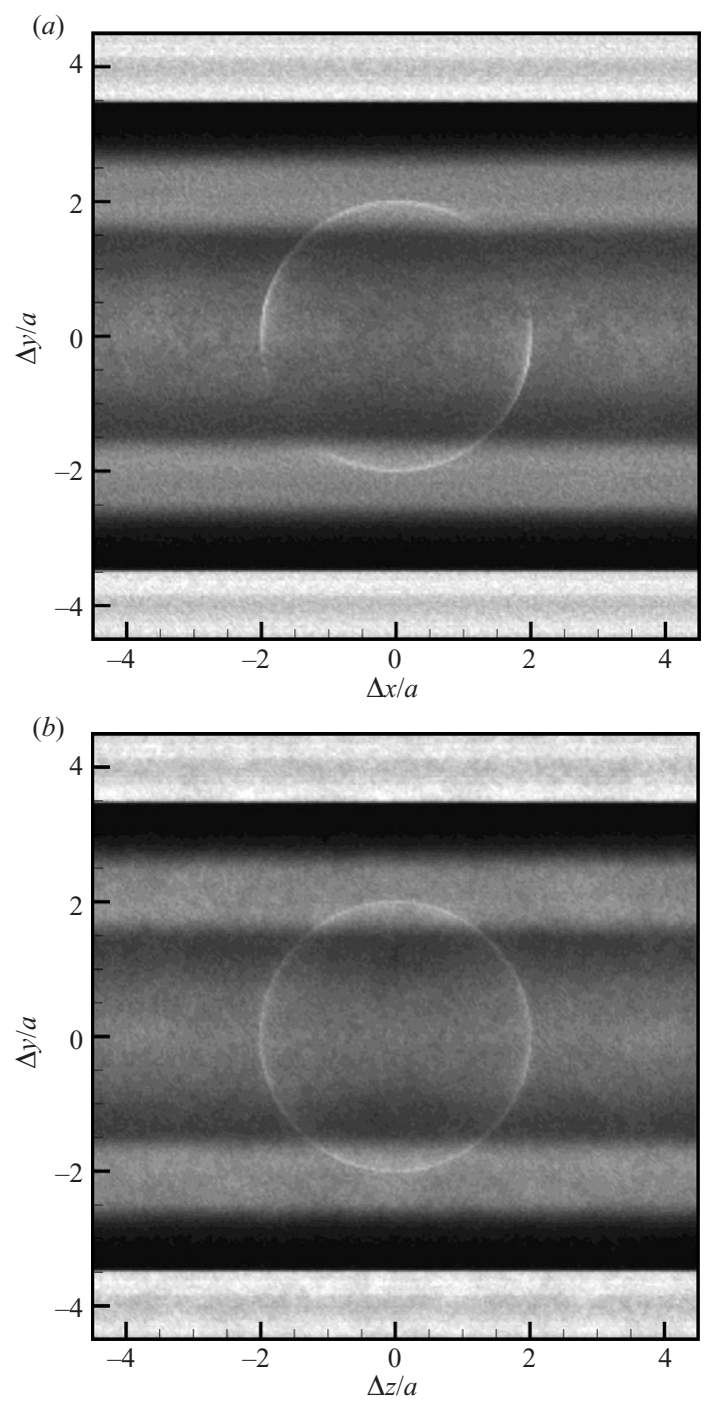

FIGURE 5. The pair-distribution function projected onto $(a) x-y$ and $(b) y-z$ planes for C4S. Light contours represent high probability.

particles centred in $4.5 \leqslant y / a \leqslant 5.5$. Two distinct streaks of high probability region are observed around $\Delta y / a \simeq \pm 2$ and \pm 4 , which correspond to the parallel particle layers at $y / a \simeq 1$ and 3 . Due to the strong layering, there is a depletion layer between two particle layers $(\Delta y / a \simeq \pm 3 \sim 3.5)$ in which the probability of finding another particle is low. In the core region $-2.5<\Delta y / a<2.5$ or $2.5<y / a<7.5, g(\Delta x, \Delta y)$ shows two shells of high probability near the compressional axis of the linear shear flow, similar to the results for sheared suspensions in an unbounded domain (Sierou \& Brady 2002).

Figure 6 shows the concentration profiles in a large channel $\left(L_{y} / a=20\right)$. The concentration profiles for $\mathrm{C} 2 \mathrm{~L}$ and $\mathrm{C} 3 \mathrm{~L}$ are similar to those in the smaller channel $\left(L_{y} / a=10\right)$. For $\phi=0.4$, the plateau of the concentration is observed when $y / a>7$. 


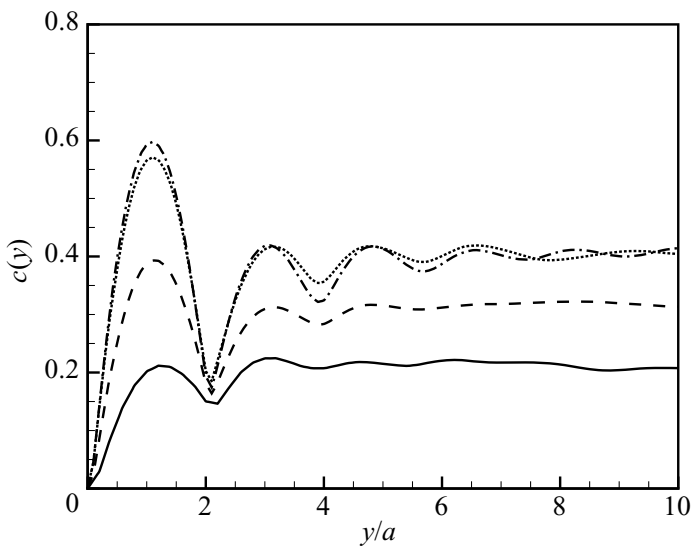

Figure 6. Concentration profiles for C2L (solid), C3L (dashed), C4La (dash-dot) and C4Lc (dotted).

In addition, to show the effect of the contact force or particle roughness, the concentration profiles for C4La and C4Lc are compared in figure 6. In C4Lc, the contact force is activated when the distance between two particles is $a \epsilon<0.001$. A very large $F_{r e f}$ is used to make the contact force behave similarly as the hard sphere potential. The minimum separation distances for C4La and C4Lc are $\epsilon_{\min } \simeq 3 \times 10^{-3}$ and $5 \times 10^{-4}$, respectively. Comparison of the profiles for C4La and C4Lc shows that indeed the specific details of the contact force has only a slight effect on the concentration profile.

The pair-distribution function $g(\Delta x, \Delta y)$ for C4La is obtained for two different regions for the reference particles; $5.5<y / a<6.5$ (figure $7 a$ ), a middle zone between the wall and the centreline, and $8<y / a<12$ (figure $7 b$ ), close to the centreline. In figure $7(a)$, three particle layers are observed in the lower half around $\Delta y / a \simeq-1.5$, -3 and -5 , which correspond to the local concentration peaks near the wall. When $\Delta y>0$, the particle layering is weak and the suspension is essentially homogeneous. Similar to suspensions in an unbounded domain, two shells of high probability region are observed oriented with the compressional axis of the mean rate of strain. Observation of the instantaneous suspension field reveals that the second and third particle layers are formed and then broken repeatedly with some period. In other words, unlike the first particle layer, the second and third particle layers exist on average not at each time instance. The pair-distribution function obtained in the core region (figure $7 b$ ) is similar to the previous results for an unbounded domain (Sierou \& Brady 2002; Yeo \& Maxey, 2010), implying the rheological properties in the core region may be explained by the results in the unbounded domain.

The concentration profiles for different $L_{y} / a$ at $\phi=0.4$ is compared in figure 8 . It is shown that, as $L_{y} / a$ increases, the peak concentration at the location of the first particle layer decreases. It is interesting to see that the concentration profiles for different $L_{y} / a$ are almost similar except for the value of the first peak, indicating the width of particle layers is independent of $L_{y} / a$, if $L_{y} / a$ is large enough. At $\phi=0.4$, the suspension field is homogeneous if $y / a>8$. This observation is consistent with the experimental result in Zarraga et al. (2000) that the rheological properties are functions of the channel width when the channel is not wide enough. As $L_{y}$ increases, the ratio of the homogeneous region to the particle layers becomes bigger, which 

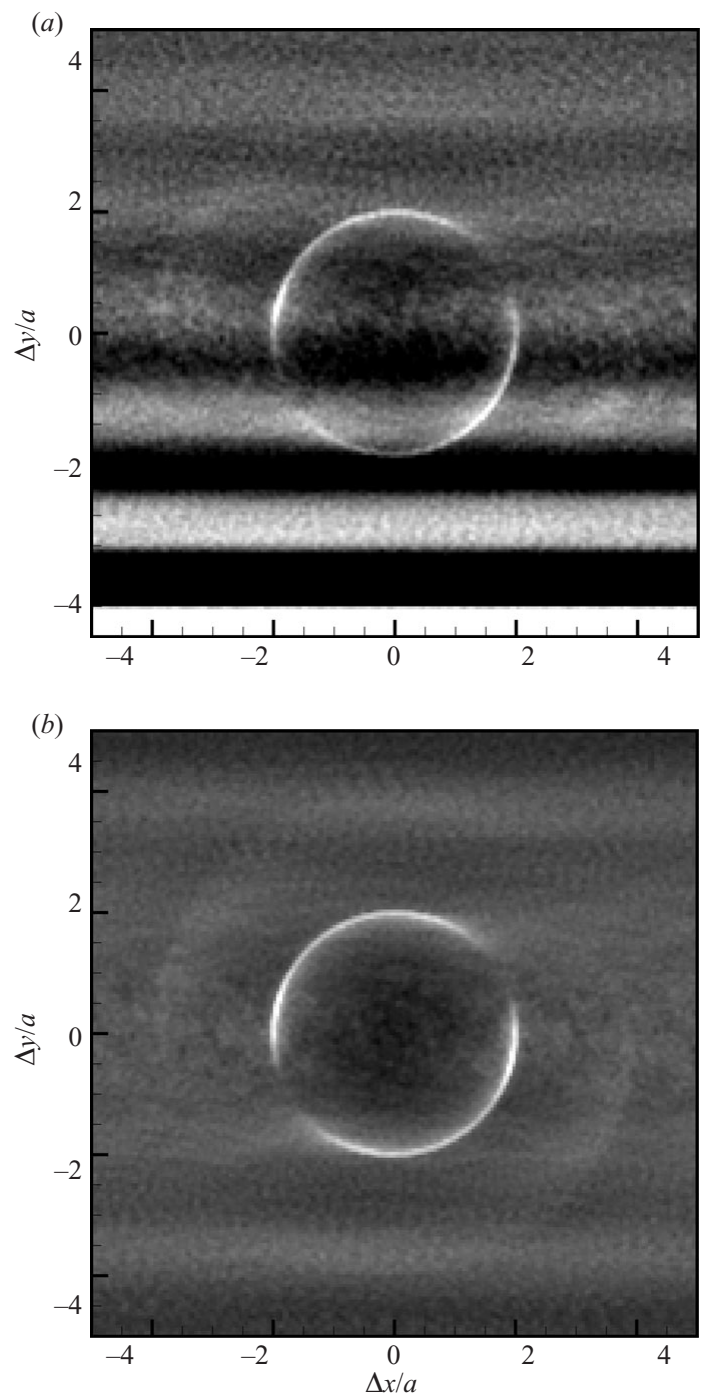

FIGURE 7. The pair-distribution functions for C4La projected onto $x-y$ plane for particles in (a) $5.5<y / a<6.5$ and (b) $8<y / a<12$. Light contours represent high probability.

makes the volume-averaged rheological properties independent of $L_{y} / a$ in a wide channel.

Similar to the concentration, the average particle flux is defined as

$$
\boldsymbol{c} \boldsymbol{v}\left(x_{2}\right)=\frac{1}{L_{x} \times L_{z}} \sum_{i=1}^{N_{p}} \iint H\left(a-\left|\boldsymbol{x}-\boldsymbol{Y}^{i}\right|\right) \boldsymbol{V}^{n} \mathrm{~d} x \mathrm{~d} z,
$$

where $H(x)$ is the Heaviside step function. Then, the averaged particle-phase velocity is obtained from

$$
\left\langle\boldsymbol{v}\left(x_{2}\right)\right\rangle=\frac{\left\langle c \boldsymbol{v}\left(x_{2}\right)\right\rangle}{\left\langle c\left(x_{2}\right)\right\rangle}
$$




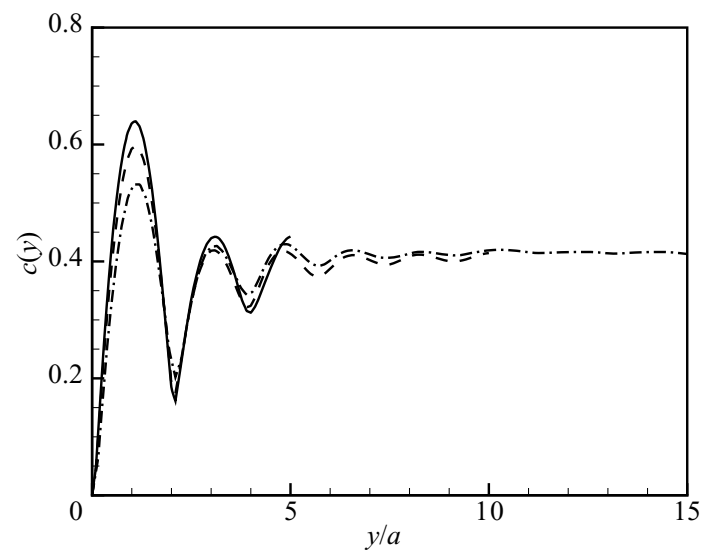

Figure 8. Concentration profiles for C4S (solid), C4La (dashed) and C4H (dash-dot).

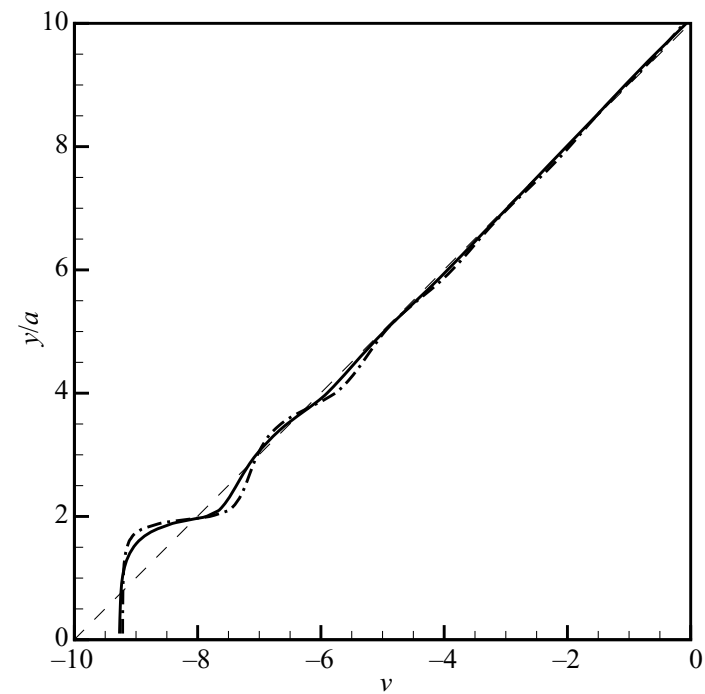

Figure 9. Average particle velocities for C3L (solid) and C4La (dash-dot). The dashed line is the linear velocity profile of Couette flow without suspensions.

The particle-phase velocity profiles for C3L and C4La are shown in figure 9. Consistent with the previous results of Singh \& Nott (2000) and Kromkamp et al. (2006), the particle-phase velocity profile is almost linear in the core region $(5<y / a<15)$. Near the wall $(y / a<0.7)$, the particle-phase velocity is faster than the linear profile, which is responsible for the apparent wall slip observed experimentally in concentrated suspension flows (Jana et al. 1995).

\subsection{Relative viscosity}

The particle contribution to the bulk stresses is given by

$$
\left\langle\sigma_{i j}^{P}\right\rangle=\frac{N_{s}}{Q^{D}}\left(\left\langle S_{i j}\right\rangle+\left\langle S_{i j}^{e}\right\rangle\right),
$$

in which $Q_{D}$ is the volume of the sampling domain over which the contribution is summed and $N_{s}$ is the number of particles in the sampling domain. The hydrodynamic 


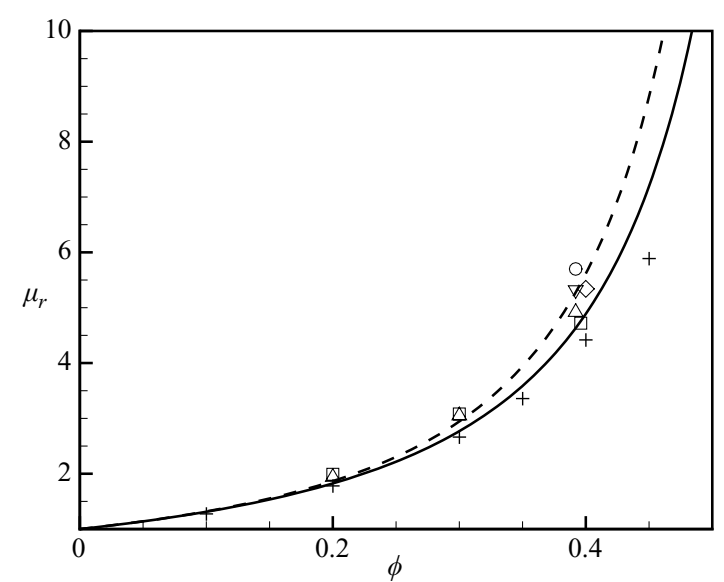

FIGURE 10. Relative viscosity $\mu_{r}$ : solid line, Krieger \& Dogherty (1959); dashed line, Eilers fit; $\square, \mathrm{C} 2 \mathrm{~S}, \mathrm{C} 3 \mathrm{~S}$ and $\mathrm{C} 4 \mathrm{~S} ; \triangle, \mathrm{C} 2 \mathrm{~L}, \mathrm{C} 3 \mathrm{~L}$ and $\mathrm{C} 4 \mathrm{La} ; \nabla, \mathrm{C} 4 \mathrm{Lb} ; \mathrm{O}, \mathrm{C} 4 \mathrm{Lc} ; \diamond, \mathrm{C} 4 \mathrm{H} ;+$, Yeo \& Maxey (2010).

$S_{i j}$ and contact-force contributions $S_{i j}^{e}$ to the total stresses are calculated by

$$
\begin{aligned}
& \left\langle S_{i j}\right\rangle=\frac{1}{N_{s}} \sum_{n=1}^{N_{s}} S_{i j}^{n}, \\
& \left\langle S_{i j}^{e}\right\rangle=\frac{1}{N_{s}} \sum_{n=1}^{N_{s}} \sum_{k=n+1}^{N_{s}}-\frac{1}{2}\left(r_{i}^{n k} F_{P, j}^{n k}+r_{j}^{n k} F_{P, i}^{n k}\right) .
\end{aligned}
$$

The relative viscosity of the suspension $\mu_{r}$ in a linear shear flow is

$$
\mu_{r}=1+\frac{1}{\mu \dot{\gamma}}\left\langle\sigma_{12}^{P}\right\rangle
$$

In figure $10, \mu_{r}$ for $Q_{D}=\left|\Omega^{D}\right|$ is compared with two viscosity models,

(a) Krieger \& Dogherty (1959),

$$
\mu_{r}=\left(1-\frac{\phi}{\phi_{m}}\right)^{-[\eta] \phi_{m}}
$$

(b) Eilers fit (Stickel \& Powell 2005),

$$
\mu_{r}=\left(1+\frac{\frac{1}{2}[\eta] \phi}{1-\phi / \phi_{m}}\right)^{2},
$$

in which $\phi_{m}$ is the maximum packing fraction and $[\eta]$ is a fitting parameter. Stickel \& Powell (2005) showed that the relevant experimental data are well represented by Eilers' fit with $[\eta]=2.5$ and $\phi_{m}=0.65$. The high-frequency dynamic viscosity $\mu_{\infty}$ in Yeo \& Maxey (2010) is obtained by the ensemble average of 100 random configurations at each $\phi$. It is shown that $\mu_{r}$ in the present simulation is in the range of previous empirical results. As microstructures form in sheared suspensions, $\mu_{r}$ is typically larger than $\mu_{\infty}$. The difference between $\mu_{r}$ and $\mu_{\infty}$, called the excess viscosity $\Delta \mu=\mu_{r}-\mu_{\infty}$, is positive and an increasing function of $\phi$ (Sierou \& Brady 2002).

Of particular note, Zarraga et al. (2000) found in their parallel plate experiments for $\phi=0.45$ that $\mu_{r}$ is an increasing function of $L_{y} / a$ when $L_{y} / a<40$ and then reaches 


$\begin{array}{lcr} & \Omega^{C} & \Omega^{W} \\ \text { C4La } & 4.18 & 3.65 \\ \text { C4H } & 4.58 & 3.86 \\ \text { Sierou \& Brady (2002) } & 4.49 \pm 0.037\end{array}$

TABLE 2. The deviatoric stress $\left\langle\sigma_{12}^{P}\right\rangle$ estimated in the core region $\Omega^{C}$ and the wall region $\Omega^{W}$.

\begin{tabular}{lllcc} 
& $R_{\text {ref }}$ & \multicolumn{1}{c}{$F_{\text {ref }}$} & $\epsilon_{\text {min }}$ & $\mu_{r}$ \\
C4La & 2.01 & 100 & $3 \times 10^{-3}$ & 4.92 \\
C4Lb & 2.004 & 100 & $1 \times 10^{-3}$ & 5.31 \\
C4Lc & 2.001 & 1000 & $5 \times 10^{-4}$ & 5.70
\end{tabular}

TABLE 3. The relative viscosity for different $R_{r e f}$ at $\phi=0.4$.

a plateau if $L_{y} / a>40$. It was suggested that the wall slip is a major mechanism responsible for the decrease in the relative viscosity (Acrivos 1993; Zarraga et al. 2000). It appears instead that the $L_{y} / a$ dependency of $\mu_{r}$ is largely due to the organized microstructure in the near-wall region (particle layering). In homogeneous concentrated suspensions, the dominant contribution to the relative viscosity comes from the normal lubrication interactions between particles, which is proportional to $\sim 1 / \epsilon$ for a gap width $a \epsilon$ (Frankel \& Acrivos 1967). On the other hand, the lubrication interactions between two particle layers is mainly through tangential motions between particles in each layers for which the singularity is $\sim \log \epsilon$. Hence, if the particle layering is dominant, the viscosity may be smaller than that in a corresponding homogeneous suspension.

These observations may be compared with the simulation results for $\mu_{r}$ at different $\phi$ and $L_{y} / a$ shown in figure 10. Since the particle layering is weak at low volume fractions, $\mu_{r}$ is less sensitive to $L_{y} / a$ when $\phi \leqslant 0.3$. On the other hand, $\mu_{r}$ is a non-decreasing function of $L_{y} / a$ at $\phi=0.4$.

The comparison may be refined by dividing the channel into zones and evaluating the average particle stresses in each zone as opposed to the whole domain $\Omega^{D}$ of the channel. Table 2 shows the deviatoric particle stress estimated from the particles in the core region $\Omega^{C}$ and wall region $\Omega^{W}$. $\Omega^{C}$ and $\Omega^{W}$ are chosen as

$$
\begin{aligned}
& \Omega^{W}=\left(0, L_{x}\right) \times\left\{\left(0, L_{w}\right) \cup\left(L_{y}-L_{w}, L_{y}\right)\right\} \times\left(0, L_{z}\right), \\
& \Omega^{C}=\Omega^{D} \backslash \Omega^{W},
\end{aligned}
$$

in which $L_{w}$ is the width of the wall region. Here, $L_{w}=5 a$ is used at $\phi=0.4$. For the comparison, the result for a homogeneous suspension from Sierou \& Brady (2002) is included. It may be seen that $\sigma_{12}^{P}$ in $\Omega^{C}$ is close to the results obtained in an unbounded domain, and further that $\sigma_{12}^{P}$ in $\Omega^{C}$ is consistently larger than that in $\Omega^{W}$. The ratios of the deviatoric stress in $\Omega^{C}$ to that in $\Omega^{W}$ are about 1.15 and 1.19 for $\mathrm{C} 4 \mathrm{La}$ and $\mathrm{C} 4 \mathrm{H}$, respectively.

Typically, $\mu_{r}$ obtained in the numerical simulations is somewhat lower than the experimental results. Sierou \& Brady (2002) argued that the discrepancy may come from the uncertainty in the contact force model. To show the effects of the contact force on rheological parameters, the relative viscosity $\mu_{r}$ at $\phi=0.4$ is computed for three different values of $R_{\text {ref }} ; R_{\text {ref }}=2.01$ (C4La), $2.004(\mathrm{C} 4 \mathrm{Lb})$ and 2.001 (C4Lc). Together with the values of $\mu_{r}$, the minimum separation distances $\epsilon_{\min }$ in each case are shown in table 3. It is apparent that $\mu_{r}$ is dependent on the choice of the contact 
force. Since the major contribution to $\mu_{r}$ from the viscous lubrication interaction is proportional to $1 / \epsilon, \mu_{r}$ increases as the minimum separation decreases. Comparing C4Lc and C4La, there is about $16 \%$ difference in $\mu_{r}$.

\subsection{Normal stresses}

In sheared suspensions of non-colloidal particles, the irreversible effects originating from any non-hydrodynamic forces will result in an anisotropic microstructure, which in turn lead to the development of the non-Newtonian stresses. Among these, the normal stresses in suspensions are of interest because of their importance in the prediction of the particle migration in inhomogeneous flows (Nott \& Brady 1994; Morris \& Boulay 1999; Miller \& Morris 2006).

The first $\left(N_{1}\right)$ and second $\left(N_{2}\right)$ normal stress differences are defined as

$$
\begin{aligned}
& N_{1}=\sigma_{11}^{P}-\sigma_{22}^{P}, \\
& N_{2}=\sigma_{22}^{P}-\sigma_{33}^{P} .
\end{aligned}
$$

Zarraga et al. (2000) measured linear combinations of the normal stresses in suspensions of non-colloidal particles. They showed that, in Couette flow, both $N_{1}$ and $N_{2}$ are negative and $\left|N_{2}\right|>\left|N_{1}\right|$ for $\phi=0.3-0.5$. Brady \& Morris (1997) showed theoretically that all three normal stress components should be compressive in a uniform shear flow. Using SD simulation, Sierou \& Brady (2002) calculated the normal stress differences for a wide range of $\phi(0.1 \leqslant \phi \leqslant 0.5)$ for a uniform shear flow. Their results show qualitatively consistent behaviour with the experimental results in Zarraga et al. (2000). However, it was observed that $\left|N_{2}\right| \simeq\left|N_{1}\right|$ in contrast to the experimental fit $\left|N_{2}\right| \simeq 3.6\left|N_{1}\right|$ found by Zarraga et al. (2000). Origin of this difference between the numerical simulations and experiments is not clear. Possible influences include the effects of the particle-particle contact force or the shearinduced migration of particles in the circular Couette flow device, which introduces curvilinear flow effects, as opposed to the simple homogeneous shear of the SD simulations. Additionally, there is the influence of the walls and the layered structures in the experiments.

Figure 11 shows the normal stress differences from the present simulations normalized by the shear stress $\tau=\mu_{r} \mu \dot{\gamma}$. For comparison, the previous numerical results from SD simulations (Sierou \& Brady 2002) are also presented. Since there is an appreciable wall effect even in the larger channels for the present simulations, a quantitative match with the previous SD simulations in an unbounded domain is not expected. Nevertheless, $N_{1}$ and $N_{2}$ in the larger domains $\left(L_{y} / a=20,30\right)$ correspond well with the SD results. At $\phi=0.4$, reducing $R_{r e f}$, the differences in $N_{1}$ and $N_{2}$ between the present simulations and Sierou \& Brady (2002) decrease.

The second normal stress difference $\left(N_{2}\right)$ calculated in the small channel $\left(L_{y} / a=10\right)$ shows a similar trend with the results in the larger domain but is larger in magnitude. On the other hand, there is a significant difference in $N_{1}$. Other SD simulations of unbounded sheared suspensions of non-colloidal particles have shown that the normal stresses are all negative and that $\left|\sigma_{11}^{P}\right|>\left|\sigma_{22}^{P}\right|>\left|\sigma_{33}^{P}\right|$ (Yurkovetsky 1997). However, in the small channel (C4S), it is found that $\left|\sigma_{11}^{P}\right| \approx\left|\sigma_{22}^{P}\right|>\left|\sigma_{33}^{P}\right|$ with all negative values. It seems that the large $N_{2} / N_{1}$ in $\mathrm{C} 4 \mathrm{~S}$ is related with the particle layering, which spans the whole channel.

The ratio of the normal stress differences, $N_{2} / N_{1}$, estimated in the present simulations are shown in figure 12 along with the SD data of Sierou \& Brady (2002). It is shown that $N_{2} / N_{1}>1$ for all the simulations while, from $\mathrm{SD}, N_{2} / N_{1}<1$. Since the normal stress is sensitive to the microstructure, the particle layers may 

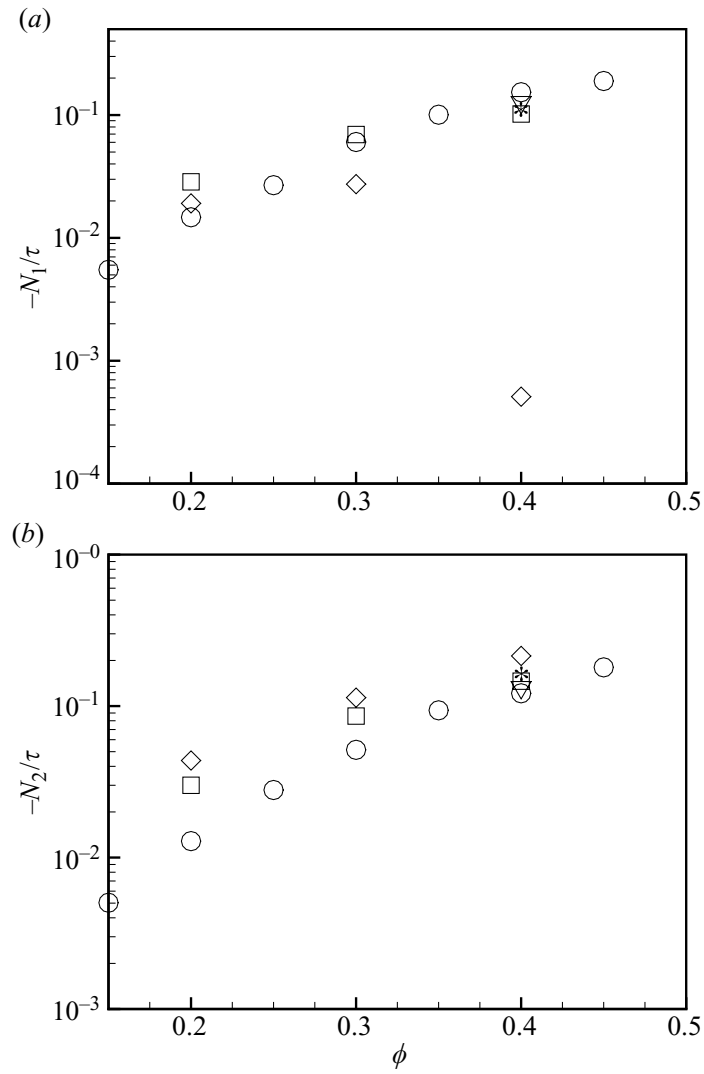

FIGURE 11. Normalized normal stress differences $(a)-N_{1} / \tau$ and $(b)-N_{2} / \tau$. $\diamond, \mathrm{C} 2 \mathrm{~S}, \mathrm{C} 3 \mathrm{~S}$ and C4S; $\square$, C2L, C3L and C4La; $\nabla$, C4Lc; *, C4H; O, Sierou \& Brady (2002).

be responsible for the difference. For example, in $\mathrm{C} 4 \mathrm{~S}$ where the wall effect is the strongest, $N_{1} \simeq 0$. To identify the effects of the walls, $N_{2} / N_{1}$ in the core $\Omega^{C}$ and the wall $\Omega^{W}$ regions are shown in figure $12(b)$, where $L_{w}=2 a$ and $3 a$ are used for $\phi=0.2$ and 0.3 , respectively, in (4.12). $N_{2} / N_{1}$ in $\Omega^{C}$ quantitatively agrees well with Sierou \& Brady (2002). On the other hand, $N_{2} / N_{1}$ estimated in $\Omega^{W}$ is larger than 3. In C2L, C3L and C4La, $1<N_{2}^{\Omega^{W}} / N_{2}^{\Omega^{C}}<1.6$ while $0.2<N_{1}^{\Omega^{W}} / N_{1}^{\Omega^{C}}<0.3$. This results suggest that the normal stresses measured in the wall-bounded domain will be different from those obtained the numerical simulation in the unbounded flows. If $L_{y} / a$ is large enough such that $\left|\Omega^{C}\right| /\left|\Omega^{W}\right| \gg 1$, it is expected that the rheological properties would be similar to those observed in an unbounded domain. However, in figure $12(a)$, it is observed that the difference between $\mathrm{C} 4 \mathrm{La}$ and $\mathrm{C} 4 \mathrm{H}$ is negligible, in which $\left|\Omega^{C}\right| /\left|\Omega^{W}\right|=1$ and 2 for $\mathrm{C} 4 \mathrm{La}$ and $\mathrm{C} 4 \mathrm{H}$, respectively. It seems that, in the largest channel used in the present simulation $\left(L_{y} / a=30\right)$, the presence of the wall has a significant effect on the suspension rheologies. As a result, it is not straightforward how to extrapolate the rheological properties for large $\left|\Omega^{C}\right| /\left|\Omega^{W}\right|$ from the present simulation results.

\subsection{Normal stresses and continuum models}

While numerical simulations based on the individual particle motion provide invaluable insight into the detailed dynamics, most of the simulation techniques are computationally too intensive to apply for engineering problems with complex 

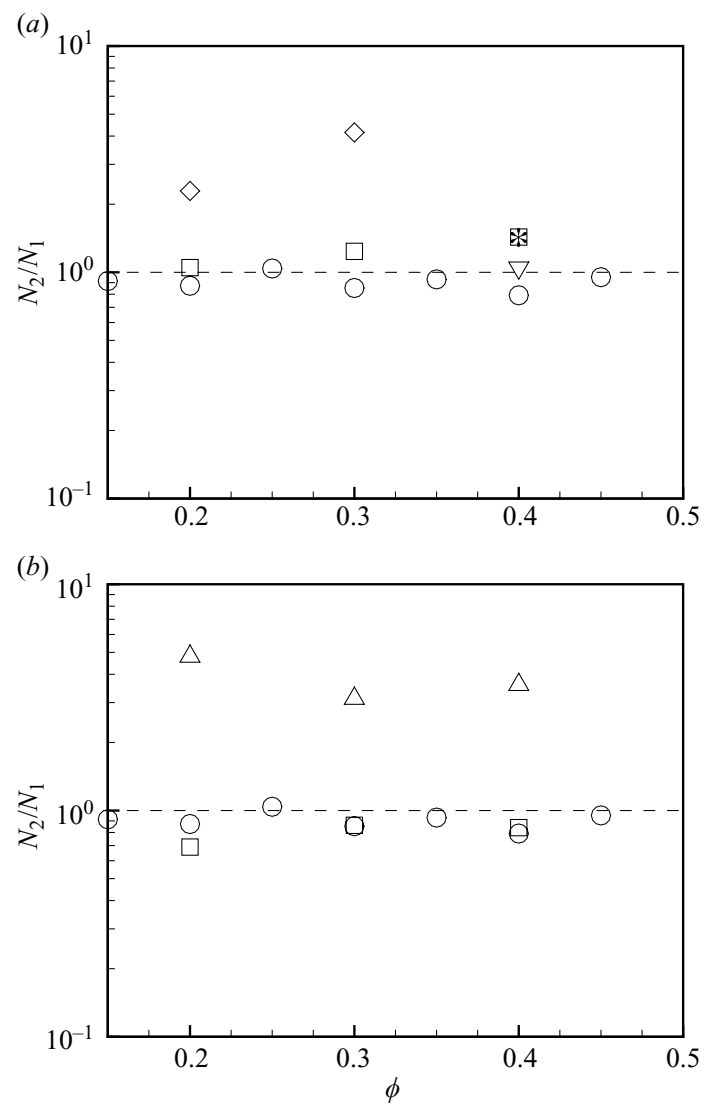

Figure 12. (a) Ratio of $N_{2}$ to $N_{1}$ in $\Omega^{D} ; \diamond, \mathrm{C} 2 \mathrm{~S}, \mathrm{C} 3 \mathrm{~S}$ and C4S; $\square$, C2L, C3L and C4La; $\nabla$, C4Lc; *, C4H. (b) Ratio of $N_{2}$ to $N_{1}$ in $\Omega^{C}(\square)$ and $\Omega^{W}(\triangle)$ for C2L, C3L and C4La. $\bigcirc$ denotes Sierou \& Brady (2002).

geometries. To predict the behaviour of the suspensions in such a flow condition, there have been several attempts to develop a continuum model (Leighton \& Acrivos 1987; Nott \& Brady 1994; Morris \& Boulay 1999; Miller \& Morris 2006; Yapici et al. 2009). One of the successful approaches is proposed by Morris \& Boulay (1999), in which the constitutive law for the particle stress for shear flows is given by

$$
\boldsymbol{\sigma}^{P}=-\mu \mu_{n}(\phi) \dot{\gamma} \boldsymbol{Q}+2 \mu \mu_{r}(\phi) \boldsymbol{e} .
$$

In this formulation, $\mu_{n}$ is the normal stress viscosity defined as

$$
\mu_{n}(\phi)=K_{n}\left(\frac{\phi / \phi_{m}}{1-\phi / \phi_{m}}\right)^{2}
$$

where $K_{n}$ is a rheological fitting parameter. The material property tensor $\boldsymbol{Q}$ is

$$
\boldsymbol{Q}=\left[\begin{array}{ccc}
1 & 0 & 0 \\
0 & \lambda_{2} & 0 \\
0 & 0 & \lambda_{3}
\end{array}\right]
$$

in which the anisotropy parameters are $\lambda_{2}=\sigma_{22}^{P} / \sigma_{11}^{P}$ and $\lambda_{3}=\sigma_{33}^{P} / \sigma_{11}^{P}$. 

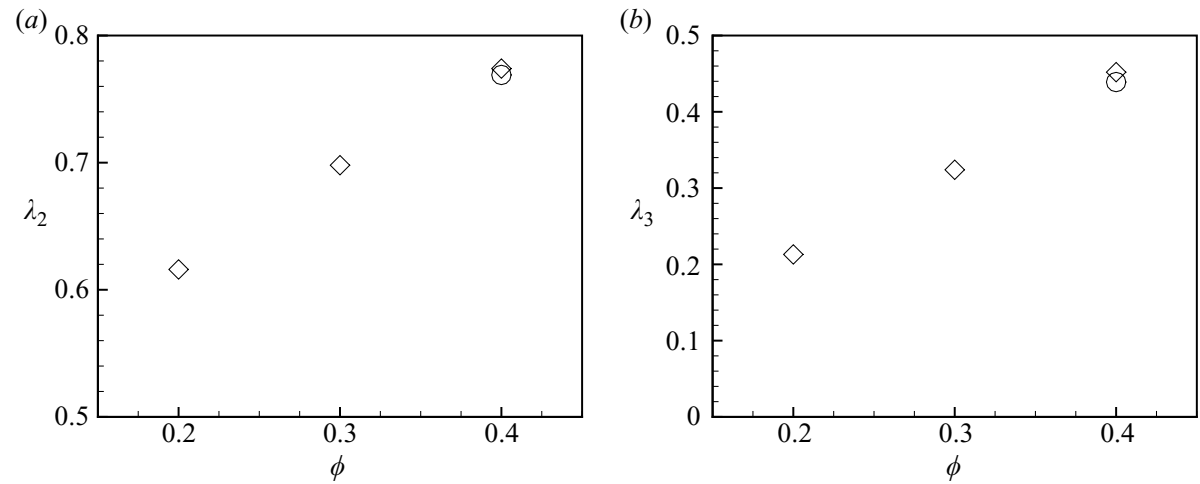

FIGURE 13. Anisotropy parameters $(a) \lambda_{2}$ and $(b) \lambda_{3} . \diamond, \mathrm{C} 2 \mathrm{~L}, \mathrm{C} 3 \mathrm{~L}$ and C4La;, $\mathrm{C} 4 \mathrm{H}$.

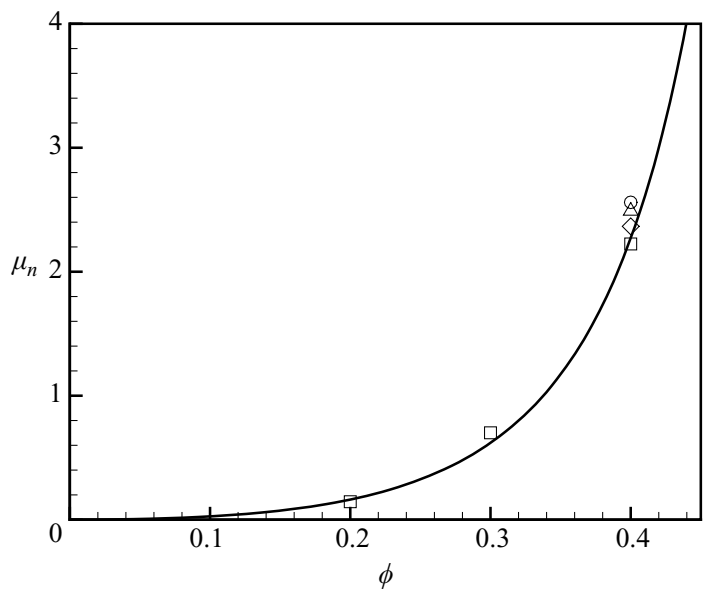

Figure 14. The normal stress viscosity in terms of $\phi . \square, \mathrm{C} 2 \mathrm{~L}, \mathrm{C} 3 \mathrm{~L}$ and $\mathrm{C} 4 \mathrm{La} ; \diamond, \mathrm{C} 4 \mathrm{Lb} ; \triangle$, C4Lc; O, C4H; - Morris \& Boulay (1999).

Morris \& Boulay (1999) suggested that $\lambda_{2} \approx 0.8$ and $\lambda_{3} \approx 0.5$ provide a good approximation to the suspension rheology and migration. Figure 13 shows the anisotropy parameters $\lambda_{2}$ and $\lambda_{3}$ as a function of $\phi$. It is shown that both $\lambda_{2}$ and $\lambda_{3}$ approach the suggested value at high $\phi$. In the experiments by Zarraga et al. (2000), a measure of the anisotropy $\left(N_{2}-N_{1}\right) / \sigma_{33}$ is an increasing function of $\phi$ for $\phi<0.4$ and then reaches a plateau. Hence, there is a possibility that both $\lambda_{2}$ and $\lambda_{3}$ become constants at higher $\phi$.

The values of $\mu_{n}$ calculated from FCM and (4.17) are shown in figure 14. $K_{n}=0.75$ and the maximum packing $\phi_{m}=0.63$ are used. The qualitative agreement of the model equation with the FCM results are satisfactory.

\subsection{Microstructure}

In this section, we investigate further the details of the microstructure using the pair distribution function $g(r, \theta, \psi)$ for C4La. Here, $\theta$ denotes the azimuthal angle measured from the flow direction (positive $x$ ) and $\psi$ is the polar angle about the $x$-axis, measured from the vorticity direction (positive $z$ ). Since most contributions to the rheological properties come from the particles near contact, the pair distribution function is shown only for $2<r / a<2$. 1 . 


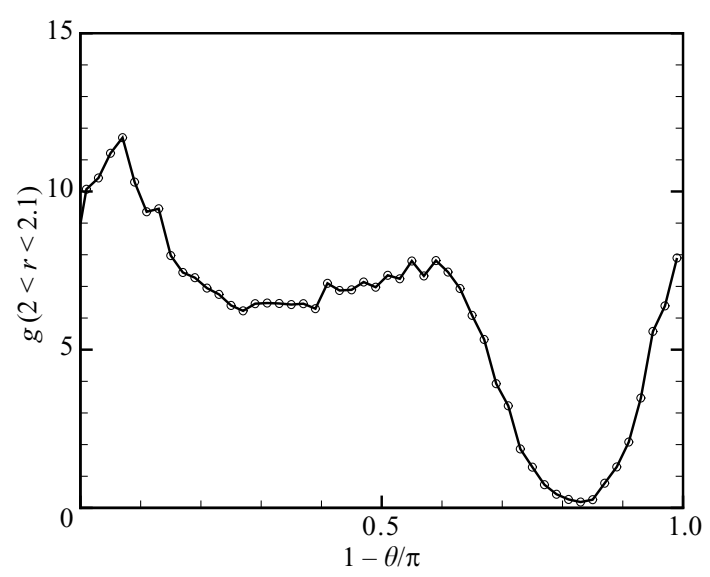

FIGURE 15. The pair distribution function for particles of which centre is located in $5 \leqslant y / a \leqslant 15$.

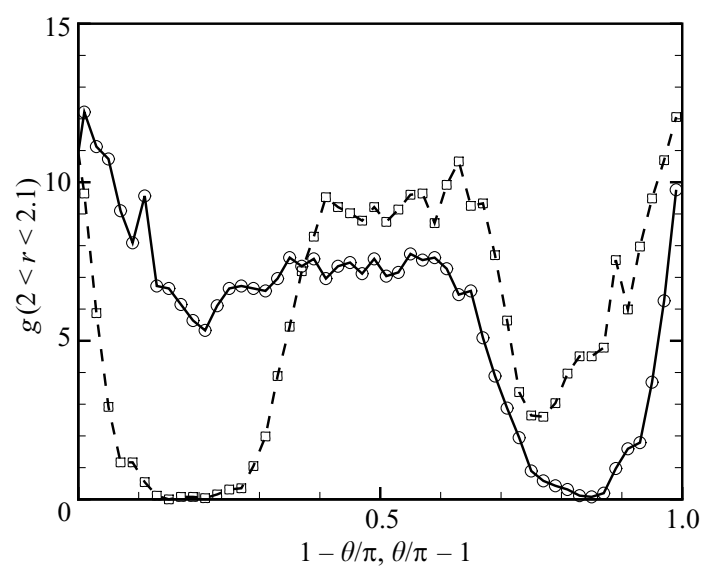

FIGURE 16. The pair distribution function for particles of which centre is located in $2 \leqslant y / a \leqslant 5$. Solid line, $1-\theta / \pi ;$ dashed line, $\theta / \pi-1$.

Figure 15 shows the pair-distribution function $g(\theta)$ in the $x-y$ plane, the plane of shear, obtained by averaging over $\pi / 2-\delta<\psi<\pi / 2+\delta$, in which $\delta=\pi / 60$, and over the reference particles whose centres are located in the core region. Considering the mirror symmetry, only $0<\theta<\pi$ is shown. It is shown that the probability to encounter another particle around the compressive axis is much higher than that in the extensive axis, which is consistent with $g(\Delta x, \Delta y)$ (figure $7 b)$. The asymmetry, which is responsible for the non-Newtonian behaviour, has been observed both in experiments (Parsi \& Gadala-Maria 1987) and in numerical simulations (Sierou \& Brady 2002; Kulkarni \& Morris 2008).

The corresponding pair-distribution function $g(\theta)$ for the reference particles centred in $2<y / a<5$ is shown in figure 16. Because the particles are in a buffer region between the strongly structured particle layer around $y / a \simeq 1$ and the free shear region (core region), the pair-distribution function is no longer mirror-symmetric. In other words, $g(\theta)$ in the upper hemisphere $0<\theta / \pi<1$ is different from the lower hemisphere $1<\theta / \pi<2$. It is observed that $g(\theta)$ in the upper hemisphere resembles that in the core region qualitatively. However, $g(\theta)$ in the lower hemisphere is distinguished by 


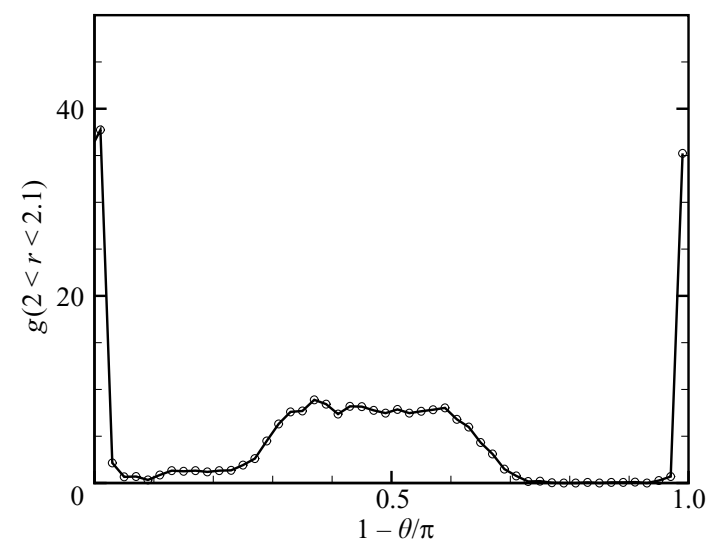

FIGURE 17. The pair distribution function for particles of which centre is located in $1 \leqslant y / a \leqslant 2$.

the increased probability near $\theta / \pi \simeq 3 / 2$, which comes from the interaction with the particle layer below.

Figure 17 shows $g(\theta)$ for the reference particles close to the wall, $1 \leqslant y / a \leqslant 2$. Distinctive peaks are observed near $\theta / \pi \simeq 0$ and 1 , indicating that it is much more probable for a particle near the wall to experience the lubrication interaction with another particle in the same particle layer than particles in another region. A plateau is observed near $\theta / \pi=0.5$ due to the interaction with the second particle layer. The location of this plateau is consistent with $g(\theta)$ for the lower hemisphere shown in figure 16 for particles centred further away from the wall. Although $g(\theta)$ is not symmetric, the asymmetry is much weaker compared to the core region (figure 15), which is responsible for the small $\left|N_{1}\right|$ in the wall region (figures 11 and 12).

\section{Conclusions}

Here, we have reported on the fully three-dimensional numerical simulations of concentrated suspensions of non-colloidal particles in Couette flow using the FCM. The FCM, with more robust lubrication corrections as given by Yeo \& Maxey (2010), is modified to incorporate the particle-wall lubrication interaction. The farfield particle-wall interaction is resolved naturally in FCM, while the particle-wall lubrication matrix is constructed for particles whose distance from a wall is less than $1.2 a$. The particle-wall lubrication correction is compared to the boundary collocation results in Ganatos et al. (1980, 1982) with good agreement.

In concentrated suspensions, the strong particle-wall lubrication inhibits particles near the wall from being resuspended into the core region. As a result, one or more particle layers are formed near the wall depending on the volume fraction. Although the present simulations are performed only for the plane Couette flow, it seems that the particle layering at high volume fraction is common in most wall-bounded flows. Even in the Poiseuille flows where most particles migrate towards the centre of the channel, there is evidence of the particle layering (Hampton et al. 1997). Since the microstructure inside of the particle layer is significantly different from that in homogeneous suspensions, it is not straightforward to predict the rheological properties in wall-bounded flows from the results obtained in unbounded domain. It is found that the width of the particle layer is not sensitive to $L_{y} / a$ once the channel 
is wide enough. At $\phi=0.4$, it is observed that the layering of particles is dominant for $0 \leqslant y / a \leqslant 7$ and beyond this the suspension is quasi-homogeneous similar to unbounded suspensions. Based on the observation, the rheological properties are calculated separately in the core and wall regions.

The rheological properties, such as the relative viscosity and normal stress differences, in the core region agree well with the results in an unbounded domain. In the wall region, the formation of particle layers leads to rheology of the suspension distinct from unbounded suspensions. As $L_{y} / a$ increases, the ratio of the core region to wall region grows larger so that the bulk rheological properties should resemble more those in unbounded suspensions. Interestingly, there is no difference in the normal stress differences between $L_{y} / a=20$ and $L_{y} / a=30$ at $\phi=0.4$ in the present simulations, even though the ratio of the core to wall region increases from 1 to 2 . It should be noted that even at $L_{y} / a=30$ it seems that suspensions are still in a discrete regime. Hence, a simple extrapolation to higher $L_{y} / a$ from the present results may not be appropriate.

The microstructure clearly shows the different dynamics depending on the distance from the wall. At $\phi=0.4$, the microstructure in the core region resembles that in the homogeneous suspensions. The shear structure (higher probability near the compressive axis) is missing in the pair-distribution function $g(\theta)$ for particles in the particle layer $(y / a<2)$. In the particle layer, most particle-particle interactions arise from the normal motion between the particles in the same particle layer $(\theta=0$ or $\pi)$ or from the tangential interaction with particles above $(\theta=\pi / 2)$. In the buffer layer $(2<y / a<5)$, the pair-distribution function $g(\theta)$ shows the characteristics of both the homogeneous suspension and the particle layer.

This work was partially supported by the NICS under TG-CTS090097 and utilized the NICS Cray XT5.

\section{Appendix. Wall resistance function}

The theoretical, asymptotic expressions for the particle-wall resistance functions can be found in the literature Corless \& Jeffrey (1988), Bossis et al. (1991) and Dance \& Maxey $(2003 a)$. Due to the typographical errors in some sources, we list the near-field forms of the resistance functions used in the simulation. As $\epsilon \rightarrow 0$, where the gap between the particle and the wall is $a \epsilon$,

$$
\begin{aligned}
& X^{A}=\frac{1}{\epsilon}-\frac{1}{5} \log \epsilon+0.8193-\frac{1}{21} \epsilon \log \epsilon, \\
& Y^{A}=-\frac{8}{15} \log \epsilon+0.9557-\frac{64}{375} \epsilon \log \epsilon, \\
& Y^{B}=\frac{2}{15} \log \epsilon+0.2568+\frac{86}{375} \epsilon \log \epsilon, \\
& X^{C}=1.2021+\frac{1}{2} \epsilon \log \epsilon, \\
& Y^{C}=-\frac{2}{5} \log \epsilon+0.3720-\frac{66}{125} \epsilon \log \epsilon, \\
& Y^{G}=-\frac{2}{3}\left(\frac{7}{10} \log \epsilon+0.923+\frac{221}{250} \epsilon \log \epsilon\right), \\
& Y^{H}=\frac{1}{10} \log \epsilon+0.923-\frac{2}{250} \epsilon \log \epsilon .
\end{aligned}
$$




\begin{tabular}{crrrrr} 
& \multicolumn{1}{c}{$C_{0}$} & \multicolumn{1}{c}{$C_{1}$} & \multicolumn{1}{c}{$C_{2}$} & \multicolumn{1}{c}{$C_{3}$} & \multicolumn{1}{c}{$C_{4}$} \\
$X^{A}$ & 8.1108 & -26.117 & 62.532 & -84.151 & \multicolumn{1}{c}{46.472} \\
$Y^{A}$ & 2.3514 & -3.6192 & 8.5516 & -13.557 & 9.8027 \\
$Y^{B}$ & -0.2295 & 1.2393 & -3.7731 & 6.6984 & -5.0789 \\
$X^{C}$ & 1.1239 & -0.3084 & 0.3610 & -0.1688 & -0.0030 \\
$Y^{C}$ & 1.4205 & -1.4217 & 3.0026 & -4.1003 & 2.6037 \\
$Y^{G}$ & 0.5629 & -1.7656 & 4.0292 & -6.1515 & 4.3758 \\
$Y^{H}$ & -0.0447 & -0.1588 & 1.0918 & -2.3320 & 1.7748
\end{tabular}

TABLE 4. FCM resistance functions.

The FCM resistance functions are given by

$$
R=C_{0}+C_{1} \epsilon+C_{2} \epsilon^{2}+C_{3} \epsilon^{3}+C_{4} \epsilon^{4} .
$$

The coefficients are shown in table 4. The particle-wall resistance function $X^{C}$ is finite at the wall, $\epsilon=0$, and is matched satisfactorily by the FCM result so that in practice, special treatment of this term is not necessary.

\section{REFERENCES}

Abbas, M., Climent, E., Simonin, O. \& MaXey, M. R. 2006 Dynamics of bidisperse suspensions under Stokes flows: linear shear flow and sedimentation. Phys. Fluids 18, 121504.

ACrivos, A. 1993 The rheology of concentrated suspensions of non-colloidal particles. In Particulate Two-Phase Flow (ed. M. C. Roco), p. 169. Butterworth-Heinemann.

BAtChelor, G. K. 1970 The stress system in a suspension of force-free particles. J. Fluid Mech. 41, 545.

Batchelor, G. K. \& Green, J. T. $1972 a$ The determination of the bulk stress in a suspension of spherical particles to order $c^{2}$. J. Fluid Mech. 56, 401.

BAtchelor, G. K. \& GreEn, J. T. $1972 b$ The hydrodynamic interaction of two small freely-moving spheres in a linear flow field. J. Fluid Mech. 56, 375.

Blake, J. R. \& Chwang, A. T. 1974 Fundamental singularities of viscous flow. J. Engng Math. 8, 23.

Bossis, G., Meunier, A. \& Sherwood, J. D. 1991 Stokesian dynamics simulations of particle trajectories near a plane. Phys. Fluids A 3, 1853.

Brady, J. F. 2001 Computer simulation of viscous suspensions. Chem. Engng Sci. 56, 2921.

Brady, J. F. \& Bossis, G. 1988 Stokesian dynamics. Annu. Rev. Fluids 20, 111.

BRADY, J. F. \& Morris, J. F. 1997 Microstructure of strongly sheared suspensions and its impact on rheology and diffusion. J. Fluid Mech. 348, 103.

Cichocki, B., Ekiel-Jezewska, M. L. \& WaJNRYB, E. 1999 Lubrication corrections for three-particle contribution to short-time self-diffusion coefficients in colloidal dispersions. J. Chem. Phys. 111, 3265 .

Corless, R. M. \& JefFrey, D. J. 1988 Stress moments of nearly touching spheres in low Reynolds number flows. J. Appl. Math. Phys. (ZAMP) 39, 874.

Da Cunha, F. R. \& Hinch, E. J. 1996 Shear-induced dispersion in a dilute suspension of rough spheres. J. Fluid Mech. 309, 211.

Dance, S. L., Climent, E. \& Maxey, M. R. 2004 Collision barrier effects on the bulk flow in a random suspension. Phys. Fluids 16, 828.

DANCE, S. L. \& MAXEY, M. R. $2003 a$ Incorporation of lubrication effects into force-coupling method for particulate two-phase flow. J. Comput. Phys. 189, 212.

Dance, S. L. \& Maxey, M. R. 2003 b Particle density stratification in transient sedimentation. Phys. Rev. E 68, 031403.

Dratler, D. I. \& Schowalter, W. R. 1996 Dynamic simulation of suspensions of non-Brownian hard spheres. J. Fluid Mech. 325, 53. 
Drazer, G., Koplik, J., Khusid, B. \& Acrivos, A. 2002 Deterministic and stochastic behaviour of non-Brownian spheres in sheared suspensions. J. Fluid Mech. 460, 307.

Durlofsky, L., Brady, J. F. \& Bossis, G. 1987 Dynamic simulation of hydrodynamically interacting particles. J. Fluid Mech. 180, 21.

EinsteIn, A. 1956 Investigations on the Theory of the Brownian Movement. Dover.

Frankel, N. A. \& ACRIVOS, A. 1967 On the viscosity of a concentrated suspension of solid spheres. Chem. Engng Sci. 22, 847.

Ganatos, P., Weinbaum, S. \& Preffer, R. 1980 A strong interaction theory for the creeping motion of a sphere between plane parallel boundaries. Part 1. Perpendicular motion. J. Fluid Mech. 99, 739.

Ganatos, P., Weinbaum, S. \& Preffer, R. 1982 Gravitational and zero-drag motion of a sphere of arbitrary size in an inclined channel at low Reynolds number. J. Fluid Mech. 124, 27.

Hampton, R. E., Mammoli, A. A., Graham, A. L., Tetlow, N. \& Altobelli, S. A. 1997 Migration of particles undergoing pressure-driven flow in a circular conduit. J. Rheol. 41, 621.

Happel, J. \& Brenner, H. 1965 Low Reynolds Number Hydrodynamics. Prentice-Hall.

Ingber, M. S., Feng, S., Graham, A. L. \& Brenner, H. 2008 The analysis of self-diffusion and migration of rough spheres in nonlinear shear flow using a traction-corrected boundary element method. J. Fluid Mech. 598, 267.

JANA, S. C., KAPOOR, B. \& ACrivos, A. 1995 Apparent wall slip velocity coefficients in concentrated suspensions of noncolloidal particles. J. Rheol. 39, 1123.

Karniadakis, G. E. \& Sherwin, S. J. 2005 Spectral/hp Element Methods for Computational Fluid Dynamics. Oxford University Press.

Kim, S. \& Karrila, S. J. 1991 Microhydrodynamics: Principles and Selected Applications. Butterworth-Heinemann.

Komnik, A., Harting, J. \& Herrmann, H. J. 2004 Transport phenomena and structuring in shear flow of suspensions near solid walls. J. Stat. Mech.: Theor. Exp., P12003.

Krieger, I. M. \& Dogherty, T. J. 1959 A mechanism for non-Newtonian flow in suspensions of rigid spheres. Trans. Soc. Rheol. 3, 137.

Kromkamp, J., van den Ende, D., Kandhai, D., van der Sman, R. \& Boom, R. 2006 Lattice Boltzmann simulation of $2 \mathrm{~d}$ and $3 \mathrm{~d}$ non-Brownian suspensions in Couette flow. Chem. Engng Sci. 61, 858.

Kulkarni, P. M. \& Morris, J. F. 2008 Suspension properties at finite Reynolds number from simulated shear flow. Phys. Fluids 20, 040602.

Leighton, D. \& ACRIVos, A. 1987 The shear-induced migration of particles in concentrated suspensions. J. Fluid Mech. 181, 415.

Liu, D., Maxey, M.R. \& Karniadakis, G.E. 2002 A fast method for particulate microflows. J. Microelectromech. Syst. 11, 691-702.

LiU, D., Maxey, M.R. \& Karniadakis, G.E. 2004 Modelling and optimization of colloidal micropumps. J. Micromech. Microengng 14, 567-575.

Lomholt, S. \& MAXEY, M. R. 2003 Force-coupling method for particulate two-phase flow: Stokes flow. J. Comput. Phys. 184, 381.

LyON, M. K. \& LEAL, L. G. 1998 An experimental study of the motion of concentrated suspensions in two-dimensional channel flow. Part 1. Monodisperse systems. J. Fluid Mech. 363, 25.

Maday, Y., Meiron, D., Patera, A. T. \& Rønquist, E. M. 1993 Analysis of iterative methods for the steady and unsteady Stokes problem: application to spectral element discretizations. SIAM J. Sci. Comput. 14, 310.

Marchioro, M. \& ACRIVOS, A. 2001 Shear-induced particle diffusivities from numerical simulations. J. Fluid Mech. 443, 101.

MaXey, M. R. \& PAtel, B. K. 2001 Localized force representations for particles sedimenting in Stokes flow. Intl J. Multiphase Flow 27, 1603-1626.

Meunier, A. \& Bossis, G. 2004 The influence of surface forces on shear-induced tracer diffusion in mono and bidisperse suspensions. Eur. Phys. J. E 506, 285.

Miller, R. M. \& Morris, J. F. 2006 Normal stress-driven migration and axial development in pressure-driven flow of concentrated suspensions. J. Non-Newton. Fluid Mech. 135, 149.

Morris, J. F. \& Boulay, F. 1999 Curvilinear flows of noncolloidal suspensions: the role of normal stresses. J. Rheol. 43, 1213. 
Nguyen, N.-Q. \& LAdD, A. J. C. 2002 Lubrication corrections for lattice-Boltzmann simulations of particle suspensions. Phys. Rev. E 66, 046708.

NotT, P. R. \& Brady, J. F. 1994 Pressure-driven flow of suspensions: simulation and theory. J. Fluid Mech. 275, 157.

Parsi, F. \& Gadala-Maria, F. 1987 Fore-and-aft asymmetry in a concentrated suspension of solid spheres. J. Rheol. 31, 725.

Sierou, A. \& Brady, J. F. 2002 Rheology and microstructure in concentrated noncolloidal suspensions. J. Rheol. 46, 1031.

Sierou, A. \& Brady, J. F. 2004 Shear-induced self-diffusion in non-colloidal suspensions. J. Fluid Mech. 506, 285.

Singh, A. \& NotT, P. R. 2000 Normal stresses and microstructure in bounded sheared suspensions via Stokesian dynamics simulations. J. Fluid Mech. 412, 279.

Smart, J. R. \& Leighton, D. T. 1989 Measurement of the hydrodynamic surface roughness of noncolloidal spheres. Phys. Fluids A 1, 52.

Stickel, J. J. \& Powell, R. L. 2005 Fluid mechanics and rheology of dense suspensions. Annu. Rev. Fluid Mech. 37, 129.

SwAN, J. W. \& BRADY, J. F. 2007 Simulation of hydrodynamically interacting particles near a no-slip boundary. Phys. Fluids 19, 113306.

Yapici, K., Powell, R. L. \& Philips, R. J. 2009 Particle migration and suspension structure in steady and oscillatory plane Poiseuille flow. Phys. Fluids 21, 053302.

YeO, K. \& MAXEY, M. R. 2010 Simulation of concentrated suspensions using the force-coupling method. J. Comput. Phys. in press.

YURKoveTSKY, Y. 1997 I. Statistical mechanics of bubbly liquids. II. Behavior of sheared suspensions of non-Brownian particles. PhD thesis, California Institute of Technology, Pasadena.

Zarraga, I. E., Hill, D. A. \& Leighton, D. T. 2000 The characterization of the total stress of concentrated suspensions of noncolloidal spheres in Newtonian fluids. J. Rheol. 44, 185.

Zurita-Gotor, M., BlawzdZiewicz, J. \& Wajnryb, E. 2007 Swapping trajectories: a new wallinduced cross-streamline particle migration mechanism in a dilute suspension of spheres. J. Fluid Mech. 592, 447. 